University of Wollongong

Research Online

Faculty of Engineering and Information

Faculty of Engineering and Information

Sciences - Papers: Part A

Sciences

2008

Differential equations and asymptotic solutions for arithmetic Asian options: 'Black-Scholes formulae' for Asian rate calls

Jeffrey Dewynne

University of Wollongong, dewynne@uow.edu.au

William Shaw

King's College, London, william.shaw@kcl.ac.uk

Follow this and additional works at: https://ro.uow.edu.au/eispapers

Part of the Engineering Commons, and the Science and Technology Studies Commons

Research Online is the open access institutional repository for the University of Wollongong. For further information contact the UOW Library: research-pubs@uow.edu.au 


\title{
Differential equations and asymptotic solutions for arithmetic Asian options: 'Black-Scholes formulae' for Asian rate calls
}

\author{
Abstract \\ In this article, we present a simplified means of pricing Asian options using partial differential equations \\ (PDEs). We first provide a concise derivation of the well-known similarity reduction and exact Laplace \\ transform solution. We then analyse the problem afresh as a power series in the volatility-scaled contract \\ duration, with a view to obtaining an asymptotic solution for the low-volatility limit, a limit which presents \\ difficulties in the context of the general Laplace transform solution. The problem is approached anew \\ from the point of view of asymptotic expansions and the results are compared with direct, high precision, \\ inversion of the Laplace transform and with numerical results obtained by V. Linetsky and J. Vecer. Our \\ asymptotic formulae are little more complicated than the standard BlackScholes formulae and, working \\ to third order in the volatility-scaled expiry, are accurate to at least four significant figures for standard \\ test problems. In the case of zero risk-neutral drift, we have the solution to fifth order and, for practical \\ purposes, the results are effectively exact. We also provide comparisons with the hybrid analytic and \\ finite-difference method of Zhang.
}

\section{Keywords}

formulae, arithmetic, solutions, asymptotic, equations, differential, scholes, black, options, asian, calls, rate

\section{Disciplines}

Engineering | Science and Technology Studies

\section{Publication Details}

Dewynne, J. N. \& Shaw, W. T. (2008). Differential equations and asymptotic solutions for arithmetic Asian options: 'Black-Scholes formulae' for Asian rate calls. European Journal of Applied Mathematics, 19 (4), 353-391. 


\title{
Differential equations and asymptotic solutions for arithmetic Asian options: 'Black-Scholes formulae' for Asian rate calls
}

\author{
J. N. DEWYNNE${ }^{1}$ and W. T. SHAW ${ }^{2}$ \\ ${ }^{1}$ OCIAM, The Mathematical Institute, 24-29 St Giles, Oxford OX1 3LB, UK \\ email: dewynne@maths.ox.ac.uk \\ ${ }^{2}$ Mathematics Department, King's College, London WC2R 2LS, UK \\ email: william.shaw@kcl.ac.uk
}

(Received 15 May 2007; revised 27 March 2008; first published online 12 May 2008)

\begin{abstract}
In this article, we present a simplified means of pricing Asian options using partial differential equations (PDEs). We first provide a concise derivation of the well-known similarity reduction and exact Laplace transform solution. We then analyse the problem afresh as a power series in the volatility-scaled contract duration, with a view to obtaining an asymptotic solution for the low-volatility limit, a limit which presents difficulties in the context of the general Laplace transform solution. The problem is approached anew from the point of view of asymptotic expansions and the results are compared with direct, high precision, inversion of the Laplace transform and with numerical results obtained by V. Linetsky and J. Vecer. Our asymptotic formulae are little more complicated than the standard Black-Scholes formulae and, working to third order in the volatility-scaled expiry, are accurate to at least four significant figures for standard test problems. In the case of zero risk-neutral drift, we have the solution to fifth order and, for practical purposes, the results are effectively exact. We also provide comparisons with the hybrid analytic and finite-difference method of Zhang.
\end{abstract}

\section{Introduction}

The subject of arithmetically averaged Asian options has been a source of interest in mathematical finance for several decades. The essential difficulty in dealing with such options is the awkward nature of the terminal distribution of a path-wise arithmetic average of an asset price which is itself log-normally distributed. A detailed probabilistic analysis for the case of an Asian rate call by Geman and Yor [4] gave, for the first time, a closed-form mathematical representation of the answer, albeit in terms of the Laplace transform of the solution. This Laplace transform involves special functions of a somewhat awkward character. The extraction of numerical results from this formula using high-level mathematical programming systems to do a direct inversion of the transform is straightforward (see e.g. Shaw [14]), but there are still computational difficulties for low volatilities. These were highlighted by $\mathrm{Fu}$ et al. [2], who further analysed the family of test cases originally introduced by Geman and Eydeland [3] that we, in common with several other authors, shall use. The analysis in [2] did not do full justice to the analysis 
given in [14] and overstated the use of Monte Carlo methods as benchmarking tools, a matter discussed in [15], but the family of test problems and general methodology of [2] has proved useful for the purposes of comparison. While the case of low volatility remains easy in Monte Carlo approaches, it continues to present difficulties in context of the analytical model of [4].

A completely different, spectral approach by Linetsky [12] gives another way of extracting accurate numerical results, and the methods of $[12,14]$, when correctly deployed, are consistent with each other and the numerical methods pioneered by Vecer [20, 21]. It should be pointed out that the results cited in [20] do resolve some difficulties in [2], notably that [2] did not correctly use the exact inversion method of [14], and perhaps placed undue emphasis on certain Monte Carlo benchmarking results that have turned out to be less accurate than the methods of [12, 14, 20, 21].

The approach of Geman and Yor, however, leaves some questions unanswered. Is it possible to give a more straightforward derivation of the solution? Can one treat the case of low volatility more effectively? Is there a continuity correction to it that allows discrete sampling of the average to be treated? We shall show that the answer to the first two of these questions is yes, and will give several options for the low-volatility situation. The issue of continuity corrections will be discussed elsewhere. Note that a simplified approach to the Geman-Yor model, based on hitting times, has also been given by Lewis [11], who also explains the use of a quadrant-based method, which is also discussed here in what we call the reduced domain approach.

Another thread of work is the reduced-form partial differential equation approach originally pioneered by Ingersoll [10]. This was developed by Rogers and Shi [13] and used by Zhang to develop a semi-analytical approximation [26]. The asymptotic expansion we present here has, as its first term, the analytical contribution to the solution noted by Zhang in his original work, [26], in which the residual component of the price is computed by a finite-difference scheme. By a more detailed formalisation of the problem in the asymptotic setting, however, we shall see how to obtain more accuracy within a purely analytical setting. Such an approach is also considered in more recent work by Zhang [27], in which he formally applies his original procedure iteratively to the residual terms in his decomposition of the price and thereby eliminates the need to numerically solve for his first residual term. This gives formal expansion in terms of functions for which explicit formulae may be computed and which appears to be equivalent to ours, but it does not explain why and where this formal series converges nor does it identify the dependence of the rate of convergence on the parameter we call $\epsilon$.

In Section 2, we derive the partial differential equations and terminal conditions that are fundamental to our approach. In Section 3, we explain how to derive the GemanYor solution using methods that are comparatively straightforward, and also relate our approach to Vecer's partial differential equation. In particular, we explain how Vecer's method may be further optimised using the reduced domain method employed to give an efficient derivation of the Geman-Yor model. In Section 4, we present an analysis based on asymptotic expansions for the average rate call. In Section 5, we obtain an asymptotic solution for the rate call based on a formal asymptotic solution of the reduced underlying risk-neutral stochastic differential equation. In Section 6, we present comparisons of results from our various approaches. The test problems we employ are a natural extension of 
the Geman-Eydeland/Fu-Madan-Wang test problems discussed in [2, 3]. Here we define analogues of their test problems to treat the cases $r=q, q>r$ in addition to problems where $r>q$ discussed in [2], and also consider variants with $\sigma \sqrt{T} \rightarrow 0$, where $r$ is the risk-free rate, $q$ the continuous dividend yield, $\sigma$ the volatility and $T$ the duration of the contract.

\section{Derivation of the fundamental PDE}

We begin by assuming that the spot price, $S_{t}$, of the asset underlying our Asian option satisfies the stochastic differential equation

$$
d S_{t}=\mu S_{t} d t+\sigma S_{t} d W_{t}
$$

where $W_{t}$ is a standard Brownian motion and $\mu$ and $\sigma$ are constants. We let

$$
I_{t}=\int_{0}^{t} w(u) S_{u} d u
$$

denote the running $w$-weighted integral of the asset price $S_{t}$.

If we let $r$ denote the constant risk-free rate, $q$ the constant continuous dividend yield, then using standard Black-Scholes dynamic hedging arguments as shown in $[22,23]$ the value, $V(S, I, t)$, of a European option which depends on $S, I$ and $t$, and has the payoff

$$
V(S, I, T)=\mathrm{P}_{o}(S, I)
$$

at expiry, $T$, satisfies the partial differential equation terminal-value problem

$$
\begin{gathered}
\frac{\partial V}{\partial t}+\frac{1}{2} \sigma^{2} S^{2} \frac{\partial^{2} V}{\partial S^{2}}+(r-q) S \frac{\partial V}{\partial S}+w(t) S \frac{\partial V}{\partial I}-r V=0 \\
V(S, I, T)=\mathrm{P}_{o}(S, I) .
\end{gathered}
$$

The solution of this problem may be shown, via the Feynman-Kac formula, to be the discounted expected value of the payoff $\mathrm{P}_{o}\left(S_{t}, I_{t}\right)$, under the risk-neutral process

$$
\begin{gathered}
d S_{t}=(r-q) S_{t} d t+\sigma S_{t} d W_{t}, \\
d I_{t}=w(t) S_{t} d t .
\end{gathered}
$$

We now carry out a series of elementary steps to simplify problem (1). One would normally expect to begin this process by removing the discounting term, $-r V$, but it turns out that this would be premature and would only have to be undone later. So we concentrate first on the essential symmetries of the problem.

\subsection{Autonomy}

We now wish to exploit the fact that (1) is autonomous in $I$. The details of how we shall use this depend on the nature of the payoff function $\mathrm{P}_{o}$. We distinguish two cases of importance. 
(1) Average-rate options, where the payoff has the form

$$
\mathrm{P}_{o}(S, I)=F_{\mathrm{r}}(I / T-K)
$$

for some function $F_{\mathrm{r}}(\cdot)$. For an average-rate call option with value $C_{\mathrm{r}}(S, I, t)$ and payoff

$$
C_{\mathrm{r}}(S, I, T)=\max \left(\frac{I}{T}-K, 0\right)
$$

we have $F_{\mathrm{rc}}(x)=\max (x, 0)$ and for an Asian-rate put option with value $P_{\mathrm{r}}(S, I, t)$ and payoff

$$
P_{\mathrm{r}}(S, I, T)=\max \left(K-\frac{I}{T}, 0\right)
$$

we have $F_{\mathrm{rp}}(x)=\max (-x, 0)$.

(2) Average-strike options, where the payoff has the form

$$
\mathrm{P}_{o}(S, I)=F_{\mathrm{s}}(S-I / T)
$$

for some function $F_{\mathrm{s}}(\cdot)$. For average-strike calls, $C_{\mathrm{s}}(S, I, t)$, the payoff is $F_{\mathrm{sc}}(x)=$ $\max (x, 0)$ and for puts, $P_{\mathrm{s}}(S, I, t)$, the payoff is $F_{\mathrm{sp}}(x)=\max (-x, 0)$.

In the first case, an average-rate option, we set $I^{\prime}=I-K T$, and in the second case we leave $I$ alone and set $I^{\prime}=I$. Regarding an option's value, $V$, as a function $V\left(S, I^{\prime}, t\right)$, the partial differential equation remains unaltered,

$$
\frac{\partial V}{\partial t}+\frac{1}{2} \sigma^{2} S^{2} \frac{\partial^{2} V}{\partial S^{2}}+(r-q) S \frac{\partial V}{\partial S}+w(t) S \frac{\partial V}{\partial I^{\prime}}-r V=0
$$

but the terminal conditions are now:

(1) average-rate call: $C_{\mathrm{r}}\left(S, I^{\prime}, T\right)=1 / T \max \left(I^{\prime}, 0\right)$;

(2) average-rate put: $P_{\mathrm{r}}\left(S, I^{\prime}, T\right)=1 / T \max \left(-I^{\prime}, 0\right)$;

(3) average-strike call: $C_{\mathrm{s}}\left(S, I^{\prime}, T\right)=\max \left(S-I^{\prime} / T, 0\right)$;

(4) average-strike put: $P_{\mathrm{s}}\left(S, I^{\prime}, T\right)=\max \left(I^{\prime} / T-S, 0\right)$.

\subsection{Scaling, dividend discounting and time reversal}

The use of reduction techniques for Asian options has been considered by many people. The first that we are aware of is the discussion of the Asian-strike case by Ingersoll [10].

Note that $V, S$ and $I^{\prime} / T$ are all prices, so (2) and the associated terminal conditions must be, and indeed are, invariant under the scaling

$$
S \rightarrow \lambda S, \quad V \rightarrow \lambda V, \quad I^{\prime} \rightarrow \lambda I^{\prime}, \quad \forall \lambda>0
$$


Two non-dimensional invariants associated with the PDE and the payoffs given above are

$$
\phi=\frac{V}{S}, \quad \eta=\frac{I^{\prime}}{S T} .
$$

Since the PDE and its terminal conditions inherit this scaling symmetry, we can reduce the problems above to ones involving only these reduced variables and time. This is equivalent to noting that, since cash does not explicitly occur in the payoffs for the calls and puts above, we can employ a change of numeraire and measure prices relative to $S$ rather than cash to simplify the problem.

It is an elementary exercise to apply the chain rule and show that $\phi$ satisfies the partial differential equation (PDE)

$$
\frac{\partial \phi}{\partial t}+\frac{1}{2} \sigma^{2} \eta^{2} \frac{\partial^{2} \phi}{\partial \eta^{2}}+\left(\frac{w(t)}{T}-(r-q) \eta\right) \frac{\partial \phi}{\partial \eta}-q \phi=0 .
$$

We now perform a further 'dividend discounting', by setting

$$
\psi=e^{-q(T-t)} \phi
$$

and we also make the usual time reversal, setting

$$
\tau_{1}=T-t \quad \text { and } \quad \mathrm{W}\left(\tau_{1}\right)=w(t)
$$

to obtain our 'master' PDE,

$$
\frac{\partial \psi}{\partial \tau_{1}}=\frac{1}{2} \sigma^{2} \eta^{2} \frac{\partial^{2} \psi}{\partial \eta^{2}}+\left(\frac{\mathrm{W}\left(\tau_{1}\right)}{T}-(r-q) \eta\right) \frac{\partial \psi}{\partial \eta} .
$$

The original terminal conditions become initial conditions in terms of $\tau_{1}$,

(1) average-rate call: $\psi(\eta, 0)=\max (\eta, 0)$;

(2) average-rate put: $\psi(\eta, 0)=\max (-\eta, 0)$;

(3) average-strike call: $\psi(\eta, 0)=\max (1-\eta, 0)$;

(4) average-strike put: $\psi(\eta, 0)=\max (\eta-1,0)$.

For average-rate options,

$$
V(S, I, t)=S e^{-q(T-t)} \psi\left(\frac{I-K T}{S T}, T-t\right)
$$

and for average-strike options,

$$
V(S, I, t)=S e^{-q(T-t)} \psi\left(\frac{I}{S T}, T-t\right) .
$$

It should be noted that our master PDE is a simple generalisation of the equation (derived by stochastic methods) of Rogers and Shi [13] to the case of non-zero (and not necessarily continuously paid) dividend yield. 


\subsection{Choices of weight function}

Different types of averaging may be achieved through various choices of the weight function $w$. We list those of most obvious interest:

(1) $w(t)=1$, which corresponds to continuous arithmetic averaging throughout the life of the instrument;

(2) $w(t)=0$ for $t<T_{1}<T$ and $w(t)=1$ for $T_{1}<t<T$, which corresponds to a continuously sampled, arithmetic average Asian tail;

(3) $w(t)=\frac{T}{N} \sum_{k=1}^{N} \delta\left(t-t_{k}\right)$, corresponding to discrete arithmetic averaging of the asset value with sampling at times $t_{k}, k=1, \ldots, N$.

\subsection{The associated SDE}

Having reduced the partial differential equation to (3), involving just one independent price-like variable, we can now appeal to the Feynman-Kac theorem to see that the solution of the PDE with the appropriate payoff is equivalent to the expected value of that payoff, where the expectation is taken with respect to the process

$$
d \eta_{t}=\left(\frac{w(t)}{T}-(r-q) \eta_{t}\right) d t+\sigma \eta_{t} d W_{t},
$$

where $W_{t}$ is a standard Brownian motion. This is perhaps the Asian problem, for payoffs with the appropriate symmetries, in its most basic and pure dimensional form. It is easy to make this the basis of a Monte Carlo simulation for European-style options. In general, there is no easy closed-form analytic formula for the terminal distribution of this process, so one must numerically integrate process paths in order to effect a Monte Carlo simulation. The terminal distribution can be obtained as a transform by twice differentiating the transform solution described in Section 3.2 with respect to the strike, though this is as hard to manage analytically as the call price. The domain of $\eta_{t}$ can be considered as the entire real line, but as noted in Section 3.1 (see also the paper by Lewis [11]), one can also restrict attention to the half line.

So far as we are aware, stochastic differential equations (SDEs) of this mixed type, which may be considered as models for a situation in which an 'asset' $(\eta)$ pays both proportional dividend yields $((r-q) \eta)$ and constant dividends $(w(t) / T)$ either continuously or discretely, were first considered by E. Wong in 1953 [24], who obtained some solutions for the transition probability density for certain signs of the parameters and with $w(t)$ constant.

\subsection{Linear solutions and parity}

We now reduce the number of cases that need to be considered by establishing the relevant parity relationships between calls and puts. As with the standard Black-Scholes problem, ${ }^{1}$ this can be treated by finding the solutions of (3) that are linear in $\eta$. Thus, we assume a

1 Like standard put-call parity, arithmetic average put-call parity is a model independent result and holds whether or not the underlying price process is geometric Brownian motion. 
solution linear in $\eta$,

$$
\psi\left(\eta, \tau_{1}\right)=a\left(\tau_{1}\right)+b\left(\tau_{1}\right) \eta
$$

and substitute into (3).

We obtain

$$
\dot{a}\left(\tau_{1}\right)+\dot{b}\left(\tau_{1}\right) \eta=\left(\frac{\mathrm{W}\left(\tau_{1}\right)}{T}-(r-q) \eta\right) b\left(\tau_{1}\right),
$$

where denotes differentiation with respect to $\tau_{1}$, and split this up into two ordinary differential equations by comparing powers of $\eta$. This gives

$$
\dot{a}\left(\tau_{1}\right)=\frac{\mathrm{W}\left(\tau_{1}\right)}{T} b\left(\tau_{1}\right), \quad \dot{b}\left(\tau_{1}\right)=-(r-q) b\left(\tau_{1}\right) .
$$

We can write the solution of these equations as

$$
a\left(\tau_{1}\right)=a(0)+b(0) f\left(\tau_{1}\right), \quad b\left(\tau_{1}\right)=b(0) e^{-(r-q) \tau_{1}},
$$

where

$$
f\left(\tau_{1}\right)=\frac{1}{T} \int_{0}^{\tau_{1}} \mathrm{~W}(s) e^{-(r-q) s} d s .
$$

It is helpful to write down the solution for $f\left(\tau_{1}\right)$ for the cases of continuous and periodically sampled discrete averaging. For continuous averaging over the life of the option, $W\left(\tau_{1}\right)=1$, we find

$$
f\left(\tau_{1}\right)=\frac{1-e^{-(r-q) \tau_{1}}}{(r-q) T},
$$

with the special case $f\left(\tau_{1}\right)=\tau_{1} / T$ when $r=q$. In the case of discrete and 'clockwork' averaging, i.e. case 3 with $t_{k}=k T / N, k=1, \ldots, N$, we set $\Delta \tau_{1}=T / N$ and let $\mathrm{C}(x)$ denote the ceiling of $x$ - the smallest integer greater than or equal to $x$. Then

$$
f\left(\tau_{1}\right)=\frac{\Delta \tau_{1}}{T} \frac{1-e^{-C\left(\tau_{1} / \Delta \tau_{1}\right)(r-q) \Delta \tau_{1}}}{1-e^{-(r-q) \Delta \tau_{1}}}
$$

with $f\left(\tau_{1}\right)=\Delta \tau_{1} \mathrm{C}\left(\tau_{1} / \Delta \tau_{1}\right)$ when $r=q$.

The parity relation for average-rate options can now be deduced by noting that, in terms of our similarity variables, a call minus a put has initial condition $\eta$ everywhere. We know the solution of the master PDE, (3), for this initial condition and deduce that the parity relation for average-rate options is

$$
\psi_{\mathrm{rc}}-\psi_{\mathrm{rp}}=f\left(\tau_{1}\right)+e^{-(r-q) \tau_{1}} \eta .
$$

For average-strike options, the initial condition for a call minus a put is just $1-\eta$, so the corresponding parity relation is

$$
\psi_{\mathrm{sc}}-\psi_{\mathrm{sp}}=1-f\left(\tau_{1}\right)-e^{-(r-q) \tau_{1}} \eta .
$$


It is a straightforward exercise to unwind these relations to those for the function $V$, and we leave this to the reader.

\section{A simple derivation of the exact solution for Asian rate calls as a Laplace transform}

From here on we focus on the case of continuous sampling where $w(t)=1$. Our master PDE, (3), serves as a convenient link to the methods developed by Geman and Yor [4] and Vecer $[20,21]$. In making the first link, we give a more straightforward derivation of the result and, in making the second link, we show how in some cases the Vecer method can be further optimised. The key to this optimisation, and the quick derivation of the Geman and Yor model, rely on the concept of reducing the domain on which the master PDE is solved.

\subsection{The reduced domain for Asian rate calls}

At first sight, it appears that the domain on which we need to solve the master PDE, (3), is $\tau_{1}>0$ and $-\infty<\eta<\infty$. This, however, is an illusion for average-rate options. One can limit attention to $\eta \leqslant 0$, for average-rate options (see also the discussion by Lewis [11]). If we return to our original representation in terms of a call then, based on the balance between $I / T$ and the strike, $K$, we see that if $I$ ever attains the value $K T$, it can never drop below that value subsequently, since it is an integral of the positive quantity $S$. That is, $I$ is non-decreasing in $t$. In terms of the quantity $\eta$, we know that if $\eta$ is positive, then the solution must be the in-the-money solution. So we can just write down the solution by noting that the payoff for positive $\eta$ is linear in $\eta$ and seeking a linear solution of the master PDE, (3), for $\eta \geqslant 0$. For a call this is just

$$
\psi_{\mathrm{rc}}\left(\eta, \tau_{1}\right)=e^{-(r-q) \tau_{1}} \eta+f\left(\tau_{1}\right)
$$

Then we note that along the at-the-money boundary, $\eta=0$, we have

$$
\psi_{\mathrm{rc}}\left(0, \tau_{1}\right)=f\left(\tau_{1}\right)
$$

So, the problem for an average-rate call is then the master PDE, (3), on the region $\eta<0$, $\tau_{1}>0$, with the boundary condition (9) and the initial condition $\psi_{\mathrm{rc}}(\eta, 0)=0$ for $\eta<0$.

\subsection{A simple derivation of the Geman-Yor formula}

We can now give a simple derivation of the Geman-Yor model for Asian call option prices [4], but see also [25]. We start with the $\mathrm{PDE}$ for $\phi$, for $\phi$ is closely related to Geman and Yor's function $C$. We introduce Geman and Yor's variables (some of their work uses $q$ for $\alpha$ and $h$ for $\tau_{2}$ );

$$
v=\frac{2(r-q)}{\sigma^{2}}-1, \quad \tau_{2}=\frac{1}{4} \sigma^{2} \tau_{1}, \quad \alpha=-\frac{1}{4} \sigma^{2} T \eta, \quad C=\frac{1}{4} \sigma^{2} e^{(r-q) \tau_{1}} T \psi .
$$


In terms of these variables, the partial differential equation (3) is

$$
\frac{\partial C}{\partial \tau_{2}}=2 \alpha^{2} \frac{\partial^{2} C}{\partial \alpha^{2}}-(1+2(v+1) \alpha) \frac{\partial C}{\partial \alpha}+2(1+v) C
$$

on the reduced domain $\alpha>0, \tau_{2}>0$ subject to the initial condition

$$
C(\alpha, 0)=0
$$

and the boundary condition

$$
C\left(0, \tau_{2}\right)=\frac{\left(e^{2(1+v) \tau_{2}}-1\right)}{2(1+v)} .
$$

We now introduce the Laplace transform

$$
\hat{C}(\alpha, p)=\int_{0}^{\infty} e^{-p \tau_{2}} C\left(\alpha, \tau_{2}\right) d \tau_{2}
$$

which satisfies the transformed equation

$$
2 \alpha^{2} \frac{\partial^{2} \hat{C}}{\partial \alpha^{2}}-(1+2(1+v) \alpha) \frac{\partial \hat{C}}{\partial \alpha}+(2(1+v)-p) \hat{C}=0
$$

with the boundary condition

$$
\hat{C}(0, p)=\frac{1}{p(p-2(1+v))} \quad \text { for } \quad \operatorname{Re}(p)>\max (2(1+v), 0),
$$

where the last restriction on $p$ ensures the transform of the boundary condition exists. The solution of this problem can be expressed in terms of a pair of confluent hyper-geometric functions

$$
\hat{C}(\alpha, p)=C_{1}(p) A_{1}(\alpha, p)+C_{2}(p) A_{2}(\alpha, p)
$$

where, in terms of $\mu=\sqrt{2 p+v^{2}}$, we have

$$
\begin{aligned}
& A_{1}(\alpha, p)=(2 \alpha)^{(2+v+\mu) / 2}{ }_{1} F_{1}\left(-\frac{1}{2}(\mu+v+2) ; 1-\mu ;-\frac{1}{2 \alpha}\right), \\
& A_{2}(\alpha, p)=(2 \alpha)^{(2+v-\mu) / 2}{ }_{1} F_{1}\left(\frac{1}{2}(\mu-v-2) ; 1+\mu ;-\frac{1}{2 \alpha}\right) .
\end{aligned}
$$

For a valid Laplace transform, we need to choose the solution that is holomorphic in a right half plane. Bearing in mind the series expansion of the hyper-geometric function (see, for example, Abramowitz and Stegun [1], Section 13.1.2), this excludes the function $A_{1}$. To establish the form of $C_{2}$, we impose the boundary condition. We note (see, for example, [1], Section 13.1.5) that if $\operatorname{Re}(z)<0$ then as $|z| \rightarrow \infty$,

$$
{ }_{1} F_{1}(a ; b ; z) \sim \frac{\Gamma(b)}{\Gamma(b-a)}(-z)^{(-a)} .
$$


Matching to the transformed boundary condition fixes $C_{2}$, and we obtain

$$
\hat{C}(\alpha, p)=\frac{(2 \alpha)^{\frac{1}{2}(v+2-\mu / 2)} \Gamma\left(2+\frac{1}{2}(\mu+v)\right)}{p(p-2(1+v)) \Gamma(1+\mu)}{ }_{1} F_{1}\left(-\frac{1}{2}(2+v-\mu) ; 1+\mu ;-\frac{1}{2 \alpha}\right) .
$$

This is the Geman and Yor model of the transformed price. In fact, Geman and Yor originally gave their result as an integral - that this integral is a hyper-geometric function is easily demonstrated and the transform $\hat{C}(\alpha, p)$ can be inverted by direct numerical integration [14]. The current version of the code of [14] is given on the web at [17]. Finally we note that for special values of $v$, the hyper-geometric functions may be simplified to linear combinations of modified Bessel functions. This is discussed briefly in Appendix A and is used later to provide benchmark numerical solutions for the case $r=q$.

\subsection{The continuous Vecer model}

As we have worked matters, we have an initial boundary value problem based on a quadrant within which the master PDE (3) holds

$$
\frac{\partial \psi}{\partial \tau_{1}}=\frac{1}{2} \sigma^{2} \eta^{2} \frac{\partial^{2} \psi}{\partial \eta^{2}}+\left(\frac{1}{T}-(r-q)\right) \frac{\partial \psi}{\partial \eta},
$$

initial condition $\psi(\eta, 0)=0$ and boundary condition $\psi\left(0, \tau_{1}\right)=f\left(\tau_{1}\right)$. Let us now make the change of independent variables

$$
t_{1}=\tau_{1}, \quad y=e^{-(r-q) \tau_{1}} \eta+f\left(\tau_{1}\right)
$$

that is, use the linear solution of the PDE as our new price-like variable. In terms of these new variables, (3) becomes

$$
\frac{\partial \psi}{\partial t_{1}}=\frac{1}{2} \sigma^{2}\left(y-f\left(t_{1}\right)\right)^{2} \frac{\partial^{2} \psi}{\partial y^{2}}
$$

This is Vecer's PDE. The initial data for a call remain $\psi(y, 0)=\max (y, 0)$. Unfortunately, either the boundary condition which was originally posed on $\eta=0$ is mapped to a condition along the time-dependent boundary $y=f\left(t_{1}\right)$, or one simply abandons the boundary condition altogether and uses the initial condition posed on the full spatial range. In the latter case, the problem becomes a pure initial value problem which, if solved numerically, involves twice the computation.

\section{Asymptotic solutions for Asian rate calls}

In this section, we consider the low-volatility limit of the problem. This is the case which is most problematic when one performs a numerical inversion of the Laplace transform of the solution derived above.

By a low-volatility limit, we mean the limit $\sigma^{2} T \ll 1$ in which the (non-dimensional) variance of final returns is small. In this limit we are able to find approximate solutions 
by the method of matched asymptotic expansions, although in our context the self-similar nature of the non-dimensional payoffs

$$
\max (\eta, 0) \text { and } \max (\eta-1,0)
$$

means that the matching conditions between inner and out solutions (see below) are satisfied automatically and need not be explicitly imposed.

The method of matched asymptotics is a well-established technique in many areas of applied mathematics; see, for example, Hinch, 1991 [6], and Van Dyke, 1978 [19]. In the context of finance, it is a less widely used technique. Howison, 2005, [7], contains a highly readable overview and examples of the application of matched asymptotic methods to option pricing problems and, more recently, Howison and co-workers $[9,8]$ have used the method to obtain quite general continuity corrections for discretely sampled European barrier and Bermudan options.

Before proceeding with the asymptotic analysis, however, we fully non-dimensionalise the problem. The semi-dimensional form of the problem is (3) with $W\left(\tau_{1}\right)=1$,

$$
\frac{\partial \psi}{\partial \tau_{1}}=\frac{1}{2} \sigma^{2} \eta^{2} \frac{\partial^{2} \psi}{\partial \eta^{2}}+\left(\frac{1}{T}-(r-q) \eta\right) \frac{\partial \psi}{\partial \eta}, \quad \tau_{1}>0, \quad-\infty<\eta<\infty
$$

subject to one of the initial conditions

$$
\begin{array}{cc}
\psi_{\mathrm{rc}}(\eta, 0)=\max (\eta, 0), & \psi_{\mathrm{rp}}(\eta, 0)=\max (-\eta, 0), \\
\psi_{\mathrm{sc}}(\eta, 0)=\max (\eta-1,0), & \psi_{\mathrm{sp}}(\eta, 0)=\max (1-\eta, 0),
\end{array}
$$

corresponding to average-rate calls and puts and average-strike calls and puts, respectively.

We introduce a non-dimensional time, based on the option's expiry date,

$$
\tau=\frac{\tau_{1}}{T}=1-\frac{t}{T}
$$

to make the problem completely non-dimensional, and introduce the non-dimensional parameters

$$
\theta=(r-q) T
$$

and

$$
\epsilon^{2}=\frac{1}{2} \sigma^{2} T
$$

In what follows, we shall assume that $\epsilon \ll 1$ and use it as the small parameter in our asymptotic series expansions.

In terms of $\tau, \theta$ and $\epsilon$, the master PDE (3) becomes

$$
\frac{\partial \psi}{\partial \tau}=\epsilon^{2} \eta^{2} \frac{\partial^{2} \psi}{\partial \eta^{2}}+(1-\theta \eta) \frac{\partial \psi}{\partial \eta}, \quad \tau>0, \quad-\infty<\eta<\infty,
$$

with the same initial conditions as given in (12). 
The critical observation is that in practice $\epsilon$ is often small and at worst, in a typical contract, of order unity. For example, for a 10-year Asian contract with $\sigma=0.5$, we have $\epsilon=1.118$ and for a two-year contract with $\sigma=0.25$, we have $\epsilon=0.25$. So for many practical purposes, we may regard $\epsilon$ as small and treat it as a perturbation parameter. The analysis which follows exploits this fact.

Given that the payoff is linear in $\eta$ as $\eta \rightarrow \pm \infty$ (and the diffusion is apparently small), it seems reasonable to assume that the solution remains linear in $\eta$, to a very high degree of approximation, as $\eta \rightarrow \pm \infty$.

Financially, this amounts to the assumption that, if the payoff is locally linear for very high and low spot prices and the option is either well in-the-money or well out-ofthe-money, then the option's $\Delta$ will be effectively a function of time only and will not significantly vary as the price of the underlying varies. That is, for a call-like payoff, if the option is deeply in-the-money, then it is going to be exercised and the holder must have the asset ready to deliver at expiry, regardless of the exact price of the asset at expiry, and likewise, if the option is well out-of-the-money it is not going to be exercised and there is no point in holding the asset. This, of course, is not strictly true as there is a small probability that, however deeply in or out-of-the-money the option is, it will change its moniness between now and expiry. This probability, however, is exponentially small for deeply in or deeply out-of-the money options. Thus, to a very high degree of approximation, the $\Gamma$ of the option is going to be zero as $\eta \rightarrow \pm \infty$ and the smaller the volatility, the better an approximation this becomes. When the option is at-the-money, however, this approximation breaks down completely because there is a high probability of the option's moniness changing between now and exercise. In this case, the diffusion and volatility terms become crucial and certainly cannot be neglected.

With this in mind, we assume an outer region where the volatility is effectively irrelevant and an inner region where it is not. The outer region, to be made more precise shortly, includes the regions $\eta \rightarrow \pm \infty$, and consists of regions where the likelihood of the option's moniness changing is negligible and the term ${ }^{2}$

$$
\epsilon^{2} \eta^{2} \frac{\partial^{2} \psi}{\partial \eta^{2}}=\mathcal{O}\left(\epsilon^{2}\right),
$$

for $\tau>0$. The inner region, also to be made more precise shortly, is essentially the complement of the outer region and consists of those regions where

$$
\epsilon^{2} \eta^{2} \frac{\partial^{2} \psi}{\partial \eta^{2}}=\mathcal{O}(1)
$$

for $\tau>0$, that is, regions where the likelihood of the option changing its moniness is significant.

For the sake of brevity, we will only present the details for an arithmetic average-rate call option; the result for the rate put follows from Asian put-call parity and the results for the strike-style options follow by a minor modification to the non-dimensional payoff and the 'critical characteristic' defined below.

2 In fact, if the payoff is linear as $\eta \rightarrow \pm \infty$, it turns out that in this region $\partial^{2} \psi / \partial \eta^{2}$ is exponentially small as a function of $\eta$. 


\subsection{The outer region and solution}

We start by assuming that if the diffusion term is multiplied by the small number $\epsilon^{2}$, then its effect will be, in the main, small. It turns out that this assumption is not uniformly valid in $\eta$, as we will find when we make an a posteriori check.

So, to begin we assume a regular perturbation solution of the form

$$
\psi(\eta, \tau)=\Phi_{0}(\eta, \tau)+\epsilon^{2} \Phi_{1}(\eta, \tau)+\cdots
$$

We find that, at leading order, the problem for $\Phi_{0}$ is the linear, first-order, hyperbolic partial differential equation ${ }^{3}$

$$
\frac{\partial \Phi_{0}}{\partial \tau}+(\theta \eta-1) \frac{\partial \Phi_{0}}{\partial \eta}=0
$$

For reasons which we will give in Section 4.2.4, there is no point in trying to determine higher order terms in this expansion: for the payoffs mentioned above, the outer solution serves chiefly to determine where the inner region lies and this is determined by the leading-order outer solution alone.

The characteristic projections for (15) are defined by

$$
\frac{d \eta}{d \tau}=(\theta \eta-1), \quad \eta(\tau=0)=\eta_{0}
$$

and are given by

$$
\eta(\tau)= \begin{cases}\eta_{0} e^{\theta \tau}+\frac{1}{\theta}\left(1-e^{\theta \tau}\right) & \theta \neq 0, \\ \eta_{0}-\tau & \theta=0 .\end{cases}
$$

Along these characteristic projections, $\Phi_{0}$ is constant ${ }^{4}$ and so if, in general,

$$
\Phi_{0}(\eta, 0)=F(\eta)
$$

then

$$
\Phi_{0}(\eta, \tau)=F\left(e^{-\theta \tau} \eta+\frac{1}{\theta}\left(1-e^{-\theta \tau}\right)\right)
$$

if $\theta \neq 0$ and

$$
\Phi_{0}(\eta, \tau)=F(\eta+\tau)
$$

if $\theta=0$.

3 This, of course, corresponds to the non-stochastic problem where the asset price grows deterministically at the rate $r-q$ and the characteristic projections are simply the non-dimensional, zero-volatility price paths.

4 Financially, this is a simple consequence of the fact that we have already factored out the risk-free discounting of the option's value at expiry in our non-dimensionalisation. 
In deriving this expansion, however, we have assumed that

$$
\epsilon^{2} \frac{\partial^{2} \Phi_{0}}{\partial \eta^{2}} \ll 1
$$

and for the payoffs we are interested in, this assumption fails along certain characteristics. Specifically, for the average-rate call, where

$$
F(\eta)=\max (\eta, 0)
$$

we find that $\partial^{2} \psi / \partial \eta^{2}$ is a delta function along the critical characteristic

$$
\eta^{*}(\tau)= \begin{cases}\frac{1}{\theta}\left(1-e^{\theta \tau}\right) & \theta \neq 0, \\ -\tau & \theta=0,\end{cases}
$$

passing at $\tau=0$ through the point of discontinuity in the slope of the payoff, ${ }^{5}$ which in this case is at the origin.

Around this critical characteristic, our regular perturbation expansion fails because along the critical characteristic

$$
\epsilon^{2} \eta^{2} \frac{\partial^{2} \psi}{\partial \eta^{2}} \gg \epsilon^{2}
$$

for $\tau>0$. Therefore, we cannot ignore the diffusion effects near this characteristic. We have to introduce a new, 'inner', region around this characteristic in which the diffusion effects balance with the drift effects.

We call the region where the regular expansion given above is valid the 'outer region'. For the average-rate call we are interested in, the outer solution is

$$
\Phi_{0}(\eta, \tau)=\max \left(\eta e^{-\theta \tau}+\frac{1}{\theta}\left(1-e^{-\theta \tau}\right), 0\right),
$$

with the obvious limit when $\theta=0$, and it turns out that it is valid in the outer region given by

$$
\left|\eta-\eta^{*}(\tau)\right| \gg \epsilon
$$

as we shall see.

Roughly speaking, this regular perturbation expansion is valid when the option is either deeply in-the-money or deeply out-of-the-money and the probability that it will change its moniness prior to expiry is negligible. In this case, the effects of volatility are unimportant (which is why we can ignore them at leading order). The critical characteristic corresponds to the option being exactly at-the-money and the inner region is the region where there is

5 For the strike-call, the critical characteristic is the characteristic projection passing, at time $\tau=0$, through the point of discontinuity in the slope of the payoff $\max (\eta-1,0)$, namely $\eta=1$, and is given by $\eta^{*}(\tau)=e^{\theta \tau}+\left(1-e^{\theta \tau}\right) / \theta$ for $\theta \neq 0$ and $\eta^{*}(\tau)=1-\tau$ for $\theta=0$. 
a significant probability that the option will change its moniness prior to expiry; here the effects of volatility are crucial and must be considered.

\subsection{The rate call with $r=q$}

The algebra involved in the analysis of the inner region is much more straightforward when $\theta=0$, i.e. $r=q$, and as discussed in Appendix A, the Laplace transform solution can also be simplified, so we shall discuss this case first in order to illustrate the principle of the method.

When $r=q, \theta=0$ and the non-dimensional form of the problem is

$$
\frac{\partial \psi}{\partial \tau}=\epsilon^{2} \eta^{2} \frac{\partial^{2} \psi}{\partial \eta^{2}}+\frac{\partial \psi}{\partial \eta}, \quad \psi(\eta, 0)=\max (\eta, 0) .
$$

The leading-order outer solution, $\Phi_{0}$, is given by

$$
\Phi_{0}(\eta, \tau)=\max (\eta+\tau, 0)
$$

and it fails near the critical characteristic

$$
\eta^{*}(\tau)=-\tau
$$

Note that the critical characteristic is defined by $(16)$, with $\eta^{*}(0)=0$.

For the inner expansion, where $\eta$ is near the critical characteristic, we introduce the scaled $^{6}$ price-like variable $\zeta$ defined by

$$
\zeta=\frac{1}{\epsilon}\left(\eta-\eta^{*}(\tau)\right) \quad \text { or } \quad \zeta=\frac{1}{\epsilon}(\eta+\tau)
$$

Applying the chain rule and using the fact that $d \eta^{*} / d \tau=-1$ when $\theta=0$ shows that this new variable eliminates the drift $\operatorname{term}^{7}$ and reduces the problem to

$$
\frac{\partial \psi}{\partial \tau}=\left(\eta^{*}(\tau)+\epsilon \zeta\right)^{2} \frac{\partial^{2} \psi}{\partial \zeta^{2}}, \quad-\infty<\zeta<\infty .
$$

We now assume a regular asymptotic series in $\epsilon$,

$$
\psi=\sum_{k=1}^{\infty} \epsilon^{k} \varphi_{k}
$$

for the inner region. In particular, in order that the series is asymptotic, we require that

$$
\left|\frac{\varphi_{k+1}(\zeta, \tau)}{\varphi_{k}(\zeta, \tau)}\right|=\mathcal{O}(1)
$$

6 If we try an inner variable of the form $\eta=\eta^{*}(\tau)+\epsilon^{\alpha} \zeta$, we find that only the choice of $\alpha=1$ leads to a problem in which time and second price-like derivatives balance in the resulting partial differential equation, i.e., $\alpha=1$ is the distinguished limit.

7 This is because the characteristic projections are paths that move with the drift and our inner variable effectively moves with one of the characteristic projections. 
for all $\tau>0$ and all $|\zeta|=\mathcal{O}(1)$. This gives the following hierarchy of partial differential equations,

$$
\begin{aligned}
& \frac{\partial \varphi_{1}}{\partial \tau}-\eta^{*}(\tau)^{2} \frac{\partial^{2} \varphi_{1}}{\partial \zeta^{2}}=0, \quad k=1 \\
& \frac{\partial \varphi_{2}}{\partial \tau}-\eta^{*}(\tau)^{2} \frac{\partial^{2} \varphi_{2}}{\partial \zeta^{2}}=2 \eta^{*}(\tau) \zeta \frac{\partial^{2} \varphi_{1}}{\partial \zeta^{2}}, \quad k=2 \\
& \frac{\partial \varphi_{k}}{\partial \tau}-\eta^{*}(\tau)^{2} \frac{\partial^{2} \varphi_{k}}{\partial \zeta^{2}}=2 \eta^{*}(\tau) \zeta \frac{\partial^{2} \varphi_{k-1}}{\partial \zeta^{2}}+\zeta^{2} \frac{\partial^{2} \varphi_{k-2}}{\partial \zeta^{2}}, \quad k>2,
\end{aligned}
$$

with the corresponding initial conditions

$$
\left.\varphi_{k}(\zeta, 0)\right)= \begin{cases}\max (\zeta, 0) & \text { if } k=1 \\ 0 & \text { if } k>1\end{cases}
$$

For the payoffs of interest it is not necessary to explicitly impose matching conditions as $\zeta \rightarrow \pm \infty$, as we shall see.

\subsubsection{Solution of first-order problem for $r=q$}

The first-order problem is

$$
\begin{gathered}
\frac{\partial \varphi_{1}}{\partial \tau}=\eta^{*}(\tau)^{2} \frac{\partial^{2} \varphi_{1}}{\partial \zeta^{2}} \\
\varphi_{1}(\zeta, 0)=\max (\zeta, 0),
\end{gathered}
$$

where $\eta^{*}(\tau)=-\tau$, and the change of variable

$$
\hat{t}(\tau)=\int_{0}^{\tau} \eta^{*}\left(\tau^{\prime}\right)^{2} d \tau^{\prime}=\frac{1}{3} \tau^{3}
$$

transforms this to

$$
\begin{gathered}
\frac{\partial \varphi_{1}}{\partial \hat{t}}=\frac{\partial^{2} \varphi_{1}}{\partial \zeta^{2}} \\
\varphi_{1}(\zeta, 0)=\max (\zeta, 0) .
\end{gathered}
$$

The solution is easily found to be

$$
\begin{aligned}
\varphi_{1}(\zeta, \tau) & =\zeta \mathrm{N}\left(\frac{\zeta}{\sqrt{2 \hat{t}}}\right)+\sqrt{\frac{\hat{t}}{\pi}} \exp \left(-\frac{\zeta^{2}}{4 \hat{t}}\right) \\
& =\zeta \mathrm{N}\left(\sqrt{\frac{3}{2 \tau^{3}}} \zeta\right)+\sqrt{\frac{\tau^{3}}{3 \pi}} \exp \left(-\frac{3 \zeta^{2}}{4 \tau^{3}}\right)
\end{aligned}
$$


where $\mathrm{N}$ is the standard normal cumulative density function

$$
\mathrm{N}(z)=\frac{1}{\sqrt{2 \pi}} \int_{-\infty}^{z} e^{-\frac{1}{2} s^{2}} d s
$$

\subsubsection{Matching the leading-order inner and outer solutions}

When $\zeta=\mathcal{O}(1 / \epsilon)$, we are moving out of the inner region and into the outer region. It is necessary to check that the inner and outer solutions agree in this region. We confirm this here. We find that

$$
\begin{aligned}
& \lim _{\zeta \rightarrow \infty} \varphi_{1}(\zeta, \tau)=\zeta+\mathcal{O}\left(e^{-\zeta^{2} / \tau^{3}}\right) \\
& \lim _{\zeta \rightarrow-\infty} \varphi_{1}(\zeta, \tau)=\mathcal{O}\left(e^{-\zeta^{2} / \tau^{3}}\right)
\end{aligned}
$$

so that for $\zeta=\mathcal{O}(1 / \epsilon)$ we have

$$
\epsilon \varphi(\zeta, \tau)= \begin{cases}\eta+\tau+\mathcal{O}\left(e^{-1 / \epsilon^{2}}\right) & \zeta>0, \\ \mathcal{O}\left(e^{-1 / \epsilon^{2}}\right) & \zeta<0\end{cases}
$$

which matches, to all algebraic orders, the outer solution

$$
\Phi_{0}(\eta, \tau)=\max (\eta+\tau, 0)
$$

\subsubsection{Solution of second-order problem for $r=q$}

The key to solving the second- and higher order problems is the observation that

$$
\frac{\partial \varphi_{1}}{\partial \zeta}=\mathrm{N}\left(\frac{\zeta}{\sqrt{2 \hat{t}}}\right)
$$

and hence that

$$
\frac{\partial^{2} \varphi_{1}}{\partial \zeta^{2}}=\frac{1}{\sqrt{4 \pi \hat{t}}} \exp \left(-\frac{\zeta^{2}}{4 \hat{t}}\right) \equiv G(\zeta, \hat{t})=G\left(\zeta, \frac{1}{3} \tau^{3}\right),
$$

where $G(\zeta, \hat{t})$ is the Green's function for the free diffusion equation in $(\zeta, \hat{t})$. Importantly, it has the properties that

$$
\frac{\partial G(\zeta, \hat{t})}{\partial \hat{t}}=\frac{\partial^{2} G(\zeta, \hat{t})}{\partial \zeta^{2}} \text { and } \frac{\partial G(\zeta, \hat{t})}{\partial \zeta}=-\frac{\zeta}{2 \hat{t}} G(\zeta, \hat{t})
$$

The problem for $\varphi_{2}$ is

$$
\frac{\partial \varphi_{2}}{\partial \tau}-\tau^{2} \frac{\partial^{2} \varphi_{2}}{\partial \zeta^{2}}=-2 \tau \zeta G\left(\zeta, \frac{1}{3} \tau^{3}\right)
$$


with

$$
\varphi_{2}(\zeta, 0)=0
$$

We seek a solution of the form

$$
\varphi_{2}(\zeta, \tau)=\zeta f(\tau) G\left(\zeta, \frac{1}{3} \tau^{3}\right)
$$

where $f$ is a function to be determined.

On the basis of this assumption and using (24), we find that

$$
\frac{\partial \varphi_{2}}{\partial \tau}-\frac{\partial^{2} \varphi_{2}}{\partial \zeta^{2}}=\left(\frac{d f}{d \tau}+\frac{3}{\tau} f\right) \zeta G(\zeta, \tau),
$$

so the PDE for $\varphi_{2}$ is satisfied iff

$$
\frac{d f}{d \tau}+\frac{3}{\tau} f=-2 \tau
$$

which gives

$$
f(\tau)=-\frac{2}{5} \tau^{2}+\frac{C_{2}}{\tau^{3}}
$$

The initial condition for $\varphi_{2}$ is automatically satisfied, since $G(\zeta, \tau) \rightarrow 0$ rapidly enough as $\tau \rightarrow 0$, no matter what the value of $C_{2}$ is. If we recall, however, that the series (20) must be asymptotic as $\tau \rightarrow 0$, then we must have $C_{2}=0$ and hence

$$
f(t)=-\frac{2}{5} \tau^{2}
$$

Thus, we are led to

$$
\begin{aligned}
\varphi_{2}(\zeta, \tau) & =-\frac{2}{5} \tau^{2} \zeta G\left(\zeta, \frac{1}{3} \tau^{3}\right) \\
& =-\frac{1}{5} \zeta \sqrt{\frac{3 \tau}{\pi}} \exp \left(-\frac{3 \zeta^{2}}{4 \tau^{3}}\right) .
\end{aligned}
$$

\subsubsection{Comments on matching and the outer solution}

We find that when $\zeta=\mathcal{O}(1 / \epsilon), \varphi_{2}(\zeta, \tau)$ is exponentially small. Thus, as we move into the outer region it vanishes to all algebraic orders. In the outer region, the corresponding outer solution, $\Phi_{1}(\eta, \tau)$, formally satisfies the same equation (15) as $\Phi_{0}$, since the second derivative of $\Phi_{0}$ is only non-zero along the critical characteristic which is inside the inner region, but with zero initial conditions. Matching it to the inner solution, which vanishes to all algebraic orders, gives us zero boundary conditions and hence the outer solution $\Phi_{1}$ vanishes. Similar results apply to all the higher order terms in the outer solution, which is why it is unnecessary to compute them. Moreover, the inner expansion $\varphi_{1}$ matches the outer solution $\Phi_{0}$ to all algebraic orders in the outer region and the outer solution is therefore effectively redundant, its only use is to determine where the inner region lies. 


\subsubsection{Solution of third-order problem}

We will see that the structure of the problem is such that the device employed to obtain the second-order solution may be used again. The PDE for $\varphi_{3}$ is given by

$$
\frac{\partial \varphi_{3}}{\partial \tau}-\tau^{2} \frac{\partial^{2} \varphi_{3}}{\partial \zeta^{2}}=-2 \tau \zeta \frac{\partial^{2} \varphi_{2}}{\partial \zeta^{2}}+\zeta^{2} \frac{\partial^{2} \varphi_{1}}{\partial \zeta^{2}} .
$$

We already know that

$$
\frac{\partial^{2} \varphi_{1}}{\partial \zeta^{2}}=G\left(\zeta, \frac{1}{3} \tau^{3}\right)
$$

and after a little calculation using (24) we find that

$$
\frac{\partial^{2} \varphi_{2}}{\partial \zeta^{2}}=-\frac{9}{10} \tau^{-2} \zeta\left(\zeta^{2}-2 \tau^{3}\right) G\left(\zeta, \frac{1}{3} \tau^{3}\right) .
$$

The PDE for $\varphi_{3}$ is then

$$
\frac{\partial \varphi_{3}}{\partial \tau}-\tau^{2} \frac{\partial^{2} \varphi_{3}}{\partial \zeta^{2}}=\left(\frac{9}{5} \tau^{-3} \zeta^{4}-\frac{13}{5} \zeta^{2}\right) G(\zeta, \tau) .
$$

As above, we make the assumption of a suitable solution, which this time involves a polynomial in $\zeta$,

$$
\varphi_{3}(\zeta, \tau)=\left(f_{1}(\tau)+f_{2}(\tau) \zeta^{2}+f_{3}(\tau) \zeta^{4}\right) G\left(\zeta, \frac{1}{3} \tau^{3}\right)
$$

Substituting this into the PDE for $\varphi_{3}$ gives the coupled system of ODEs,

$$
\begin{aligned}
\frac{d f_{1}(\tau)}{d \tau}-2 \tau^{2} f_{2}(\tau) & =0 \\
\frac{d f_{2}(\tau)}{d \tau}+\frac{6}{\tau} f_{2}(\tau)-12 \tau^{2} f_{3}(\tau) & =-\frac{13}{5} \\
\frac{d f_{3}(\tau)}{d \tau}+\frac{12}{\tau} f_{3}(\tau) & =\frac{9}{5} \tau^{-3}
\end{aligned}
$$

These may be solved in sequence using integrating factors to give

$$
\begin{aligned}
& f_{1}(\tau)=-\frac{11}{350} \tau^{4}, \\
& f_{2}(\tau)=-\frac{11}{175} \tau, \\
& f_{3}(\tau)=\frac{9}{50} \tau^{-2},
\end{aligned}
$$

where again all arbitrary constants have been set to zero as they lead to stronger singularities in these functions which, in turn, violate the asymptotic condition on (20).

Note that, again, this term in the solution is exponentially small when $\zeta=\mathcal{O}(1 / \epsilon)$. 
4.2.6 The general higher order term for $r=q$

If, for $k>1$, we make the assumption that

$$
\varphi_{k}=f_{k}(\zeta, \tau) G\left(\zeta, \frac{1}{3} \tau^{3}\right)
$$

then

$$
\begin{aligned}
& f_{2}(\zeta, \tau)=-\frac{2}{5} \tau^{2} \zeta, \\
& f_{3}(\zeta, \tau)=-\frac{11}{350} \tau^{4}-\frac{11}{175} \tau^{2} \zeta^{2}+\frac{9}{50} \tau^{-2} \zeta^{4},
\end{aligned}
$$

where again, the condition that the series (20) be asymptotic translates into the condition that $f_{2}$ and $f_{3}$ have the weakest possible singularities at $\tau=0$.

After some work we find that, for $k>3, f_{k}$ must satisfy the recursive system of partial differential equations

$$
\begin{aligned}
\frac{\partial f_{k}}{\partial \tau}-\tau^{2} \frac{\partial^{2} f_{k}}{\partial \zeta^{2}}+\frac{3 \zeta}{\tau} \frac{\partial f_{k}}{\partial \zeta}= & -2 \tau \zeta \frac{\partial^{2} f_{k-1}}{\partial \zeta^{2}}+\frac{6 \zeta^{2}}{\tau^{2}} \frac{\partial f_{k-1}}{\partial \zeta}+\left(\frac{3 \zeta}{\tau^{2}}-\frac{9 \zeta^{3}}{2 \tau^{5}}\right) f_{k-1} \\
& +\zeta^{2} \frac{\partial^{2} f_{k-2}}{\partial \zeta^{2}}-\frac{3 \zeta^{3}}{\tau^{3}} \frac{\partial f_{k-2}}{\partial \zeta}+\left(\frac{9 \zeta^{4}}{4 \tau^{6}}-\frac{3 \zeta^{2}}{2 \tau^{3}}\right) f_{k-2}
\end{aligned}
$$

Further solutions of this sequence of PDEs may, in principle, be found as polynomials in $\zeta$ whose coefficients satisfy ordinary differential equations in $\tau$. Working with this scheme ${ }^{8}$ gives an equation for $f_{4}$, for example,

$$
\frac{\partial f_{4}}{\partial \tau}-\tau^{2} \frac{\partial^{2} f_{4}}{\partial \zeta^{2}}+\frac{3 \zeta}{\tau} \frac{\partial f_{4}}{\partial \zeta}=-\frac{81}{100} \frac{\zeta^{7}}{\tau^{7}}+\frac{297}{70} \frac{\zeta^{5}}{\tau^{4}}-\frac{93}{28} \frac{\zeta^{3}}{\tau}+\frac{11}{70} \tau^{2} \zeta
$$

with the (least singular) solution

$$
f_{4}=-\frac{27}{500} \frac{\zeta^{7}}{\tau^{6}}+\frac{144}{875} \frac{\zeta^{5}}{\tau^{3}}-\frac{1}{300} \zeta^{3}+\frac{4}{175} \tau^{3} \zeta .
$$

Similarly, the (least singular) fifth-order solution can be shown to be

$$
f_{5}=\frac{243}{20000} \frac{\zeta^{10}}{\tau^{10}}-\frac{1377}{14000} \frac{\zeta^{8}}{\tau^{7}}+\frac{13317}{98000} \frac{\zeta^{6}}{\tau^{4}}-\frac{29}{2695} \frac{\zeta^{4}}{\tau}+\frac{2049}{431200} \tau^{2} \zeta^{2}+\frac{2049}{1078000} \tau^{5} .
$$

Although more terms may be found, those given above more than suffice for our purposes. They are all exponentially small outside the inner region.

\subsubsection{Summary complete to fifth order for $r=q$}

For the purposes of numerical evaluation, it is convenient to gather together the following summary of the first five terms in our asymptotic solution for $r=q$. Firstly, $\tau, \epsilon$ and $\hat{t}$ are

8 The fourth- and fifth-order terms were computed using the symbolic capabilities of Mathematica. 
defined in terms of the original, financial, variables by

$$
\tau=\frac{T-t}{T}, \quad \epsilon^{2}=\frac{1}{2} \sigma^{2} T, \quad \hat{t}=\frac{1}{3} \tau^{3}=\frac{(T-t)^{3}}{3 T^{3}}
$$

and the Green's function for the diffusion equation in $(\zeta, \hat{t})$ is

$$
G(\zeta, \hat{t})=\frac{1}{\sqrt{4 \pi \hat{t}}} \exp \left(-\frac{\zeta^{2}}{4 \hat{t}}\right)
$$

Our inner asymptotic solution for the non-dimensional problem (14) is

$$
\psi(\zeta, \tau)=\epsilon \varphi_{1}(\zeta, \tau)+\epsilon^{2} \varphi_{2}(\zeta, \tau)+\epsilon^{3} \varphi_{3}(\zeta, \tau)+\epsilon^{4} \varphi_{4}(\zeta, \tau)+\epsilon^{5} \varphi_{5}(\zeta, \tau)+\cdots
$$

and the first five terms in this series, for $r=q$ and $\theta=0$, are given by

$$
\begin{aligned}
& \varphi_{1}=\zeta \mathrm{N}\left(\frac{\zeta}{\sqrt{2 \hat{t}}}\right)+2 \hat{t} G(\zeta, \hat{t}) \\
& =\zeta \mathrm{N}\left(\sqrt{\frac{3}{2 \tau^{3}} \zeta}\right)+\sqrt{\frac{\tau^{3}}{3 \pi}} \exp \left(-\frac{3 \zeta^{2}}{4 \tau^{3}}\right) \\
& \varphi_{2}=-\frac{2}{5} \tau^{2} \zeta G(\zeta, \hat{t}) \\
& =-\frac{1}{5} \sqrt{\frac{3 \tau}{\pi}} \zeta \exp \left(-\frac{3 \zeta^{2}}{4 \tau^{3}}\right) \\
& \varphi_{3}=\left(-\frac{11}{350} \tau^{4}-\frac{11}{175} \tau \zeta^{2}+\frac{9}{50} \frac{\zeta^{4}}{\tau^{2}}\right) G(\zeta, \hat{t}) \\
& =\left(-\frac{11}{350} \tau^{4}-\frac{11}{175} \tau \zeta^{2}+\frac{9}{50} \frac{\zeta^{4}}{\tau^{2}}\right) \sqrt{\frac{3}{4 \pi \tau^{3}}} \exp \left(-\frac{3 \zeta^{2}}{4 \tau^{3}}\right), \\
& \varphi_{4}=\left(-\frac{27}{500} \frac{\zeta^{7}}{\tau^{6}}+\frac{144}{875} \frac{\zeta^{5}}{\tau^{3}}-\frac{1}{300} \zeta^{3}+\frac{4}{175} \tau^{3} \zeta\right) G(\zeta, \hat{t}), \\
& \varphi_{5}=\left(\frac{243}{20000} \frac{\zeta^{10}}{\tau^{10}}-\frac{1377}{14000} \frac{\zeta^{8}}{\tau^{7}}+\frac{13317}{98000} \frac{\zeta^{6}}{\tau^{4}}-\frac{29}{2695} \frac{\zeta^{4}}{\tau}+\frac{2049}{431200} \tau^{2} \zeta^{2}+\frac{2049}{1078000} \tau^{5}\right) G(\zeta, \hat{t}) .
\end{aligned}
$$

It turns out that for many of our test problems, with parameters of practical interest, the fifth-order solution is essentially exact, agreeing, for example, with the contour integral solution to six significant figures. Four significant figure accuracy is achievable if we keep terms up to and including $\varphi_{3}$. 


\subsection{The general case: $r \neq q$}

When $r \neq q$, the non-dimensional problem for a rate call is

$$
\begin{gathered}
\frac{\partial \psi}{\partial \tau}=\epsilon^{2} \eta^{2} \frac{\partial^{2} \psi}{\partial \eta^{2}}+(1-\theta \eta) \frac{\partial \psi}{\partial \eta}, \quad \tau>0, \quad-\infty<\eta<\infty, \\
\psi(\eta, 0)=\max (\eta, 0), \quad-\infty<\eta<\infty .
\end{gathered}
$$

The leading-order outer solution is given by (18) and it also is only valid for $\left|\eta-\eta^{*}(\tau)\right| \gg \epsilon$. To find the inner expansion, we again use the inner variable $\zeta$, defined by

$$
\zeta=\frac{1}{\epsilon}\left(\eta-\eta^{*}(\tau)\right)
$$

where in this case $\eta^{*}(\tau)$ is defined by

$$
\frac{d \eta^{*}}{d \tau}=\theta \eta^{*}-1, \quad \eta^{*}(0)=0
$$

and given by (17),

$$
\eta^{*}(\tau)=\frac{1}{\theta}\left(1-e^{\theta \tau}\right)
$$

Applying the chain rule and using (29) which defines the critical characteristic, we find that in terms of $\zeta$ and $\tau$ the problem simplifies to

$$
\begin{gathered}
\frac{\partial \psi}{\partial \tau}=\left(\eta^{*}(\tau)+\epsilon \zeta\right)^{2} \frac{\partial^{2} \psi}{\partial \zeta^{2}}-\theta \zeta \frac{\partial \psi}{\partial \zeta}, \quad \tau>0, \quad-\infty<\zeta<\infty \\
\psi(\zeta, 0)=\epsilon \max (\zeta, 0), \quad-\infty<\zeta<\infty .
\end{gathered}
$$

We again assume the regular perturbation expansion (20),

$$
\psi=\sum_{k=1}^{\infty} \epsilon^{k} \varphi_{k}
$$

and obtain the hierarchy of PDEs,

$$
\begin{aligned}
& \frac{\partial \varphi_{1}}{\partial \tau}-\eta^{*}(\tau)^{2} \frac{\partial^{2} \varphi_{1}}{\partial \zeta^{2}}+\theta \zeta \frac{\partial \varphi_{1}}{\partial \zeta}=0, \quad k=1, \\
& \frac{\partial \varphi_{2}}{\partial \tau}-\eta^{*}(\tau)^{2} \frac{\partial^{2} \varphi_{2}}{\partial \zeta^{2}}+\theta \zeta \frac{\partial \varphi_{2}}{\partial \zeta}=2 \eta^{*}(\tau) \zeta \frac{\partial^{2} \varphi_{1}}{\partial \zeta^{2}}, \quad k=2, \\
& \frac{\partial \varphi_{k}}{\partial \tau}-\eta^{*}(\tau)^{2} \frac{\partial^{2} \varphi_{k}}{\partial \zeta^{2}}+\theta \zeta \frac{\partial \varphi_{k}}{\partial \zeta}=2 \eta^{*}(\tau) \zeta \frac{\partial^{2} \varphi_{k-1}}{\partial \zeta^{2}}+\zeta^{2} \frac{\partial^{2} \varphi_{k-2}}{\partial \zeta^{2}}, \quad k>2
\end{aligned}
$$

with the respective initial conditions

$$
\varphi_{k}(\zeta, 0)= \begin{cases}\max (\zeta, 0) & \text { if } k=1 \\ 0 & \text { if } k>1\end{cases}
$$


4.3.1 Solution of first-order problem for $r \neq q$

The problem for the first-order term is

$$
\begin{gathered}
\frac{\partial \varphi_{1}}{\partial \tau}=\eta^{*}(\tau)^{2} \frac{\partial^{2} \varphi_{1}}{\partial \zeta^{2}}-\theta \zeta \frac{\partial \varphi_{1}}{\partial \zeta}, \\
\varphi_{1}(\zeta, 0)=\max (\zeta, 0) .
\end{gathered}
$$

To solve it, we introduce the new variables

$$
\hat{t}=\hat{t}(\tau), \quad \text { and } \quad x=\hat{f}(\tau) \zeta
$$

where $\hat{t}(\tau)$ and $\hat{f}(\tau)$ are yet to be determined. From the chain rule, we have

$$
\frac{\partial}{\partial \tau}=\frac{d \hat{t}}{d \tau} \frac{\partial}{\partial t}+\zeta \frac{d \hat{f}}{d \tau} \frac{\partial}{\partial x} \quad \text { and } \quad \frac{\partial}{\partial \zeta}=\hat{f}(\tau) \frac{\partial}{\partial x} .
$$

The PDE for first-order term is then

$$
\frac{d \hat{t}}{d \tau} \frac{\partial \varphi_{1}}{\partial \hat{t}}=\eta^{*}(\tau)^{2} \hat{f}(\tau)^{2} \frac{\partial^{2} \varphi_{1}}{\partial x^{2}}-\zeta\left(\frac{d \hat{f}}{d \tau}+\theta \hat{f}\right) \frac{\partial \varphi_{1}}{\partial x}
$$

and we can reduce this to the pure diffusion equation, in $(x, \hat{t})$, by choosing

$$
\frac{d \hat{f}}{d \tau}+\theta \hat{f}=0 \quad \text { and } \quad \frac{d \hat{t}}{d \tau}=\left(\eta^{*}(\tau) \hat{f}(\tau)\right)^{2} .
$$

Further, if we insist that

$$
\hat{f}(0)=1, \quad \hat{t}(0)=0
$$

then, apart from replacing $\zeta$ by $x$, the initial condition remains unaltered. We find that this implies that

$$
\begin{aligned}
\hat{f}(\tau) & =e^{-\theta \tau}, \\
\hat{t}(\tau) & =\frac{4 e^{-\theta \tau}-e^{-2 \theta \tau}+2 \theta \tau-3}{2 \theta^{3}} \\
& =\frac{1}{3} \tau^{3}-\frac{1}{4} \theta \tau^{4}+\frac{7}{60} \theta^{2} \tau^{5}-\frac{1}{24} \theta^{3} \tau^{6}+\frac{31}{2520} \theta^{4} \tau^{7}-\frac{1}{320} \theta^{5} \tau^{8}+\mathcal{O}\left(\theta^{6} \tau^{9}\right),
\end{aligned}
$$

the series expansion showing that that our scaled time is consistent with the $\theta=0$ case and also being useful for numerical evaluation when $\theta \tau \ll 1$ (see below).

We may now solve the diffusion equation

$$
\frac{\partial \varphi_{1}}{\partial \hat{t}}=\frac{\partial^{2} \varphi_{1}}{\partial x^{2}}
$$

subject to the initial condition

$$
\varphi_{1}(x, 0)=\max (x, 0)
$$


to find that in this case,

$$
\varphi_{1}=x \mathrm{~N}\left(\frac{x}{\sqrt{2 \hat{t}(\tau)}}\right)+\sqrt{\frac{\hat{t}(\tau)}{\pi}} \exp \left(-\frac{x^{2}}{4 \hat{t}(\tau)}\right)
$$

where $\mathrm{N}(\cdot)$ is the normal cumulative density. In this case, it is clearly more concise to leave $\varphi_{1}$ expressed in terms of $x$ and $\hat{t}(\tau)$.

Note that as we let $\zeta \rightarrow \pm \infty$, we recover the outer solution to all algebraic orders.

As before, it is important that

$$
\frac{\partial^{2} \varphi_{1}}{\partial x^{2}}=\frac{1}{\sqrt{4 \pi \hat{t}(\tau)}} \exp \left(-\frac{x^{2}}{4 \hat{t}(\tau)}\right) \equiv G(x, \hat{t}(\tau)) .
$$

\subsubsection{Solution of second-order problem for $r \neq q$}

The problem for $\varphi_{2}$ is

$$
\begin{gathered}
\frac{\partial \varphi_{2}}{\partial \tau}-\eta^{*}(\tau)^{2} \frac{\partial^{2} \varphi_{2}}{\partial \zeta^{2}}+\theta \zeta \frac{\partial \varphi_{2}}{\partial \zeta}=2 \eta^{*}(\tau) \zeta \frac{\partial^{2} \varphi_{1}}{\partial \zeta^{2}}, \\
\varphi_{2}(\zeta, 0)=0
\end{gathered}
$$

and, in terms of $x$ and $\hat{t}$, it becomes

$$
\begin{gathered}
\frac{\partial \varphi_{2}}{\partial \hat{t}}-\frac{\partial^{2} \varphi_{2}}{\partial x^{2}}=\frac{2}{\eta^{*}(\tau) e^{-\theta \tau}} x G(x, \hat{t}), \\
\varphi_{2}(x, 0)=0 .
\end{gathered}
$$

We proceed in a manner similar to the case $r=q$, and seek a solution in the form

$$
\varphi_{2}(x, \hat{t})=x g(\hat{t}) G(x, \hat{t})
$$

where $g(\hat{t})$ has the lowest possible singularity as $\hat{t} \rightarrow 0$. On this assumption we have

$$
\frac{\partial \varphi_{2}}{\partial \hat{t}}-\frac{\partial^{2} \varphi_{2}}{\partial x^{2}}=\left(\frac{d g}{d \hat{t}}+\frac{1}{\hat{t}} g\right) x G(x, \hat{t}),
$$

so the PDE is satisfied iff

$$
\frac{d g}{d \hat{t}}+\frac{1}{\hat{t}} g=\frac{2}{\eta^{*}(\tau) e^{-\theta \tau}}
$$

To solve this ODE for $g(\hat{t})$, let $h(\tau)=\hat{t}(\tau) g(\tau)$ and revert to $\tau$ as an independent variable. The ODE for $h$ is then

$$
\frac{d h}{d \tau}=\frac{d h}{d \hat{t}} \frac{d \hat{t}}{d \tau}=\frac{d h}{d \hat{t}}\left(\eta^{*}(\tau) e^{-\theta \tau}\right)^{2}=2 \hat{t}(\tau) \eta^{*}(\tau) e^{-\theta \tau}
$$


and hence may be integrated, with the initial condition that $h(0)=0$, to give

$$
h(\tau)=\frac{e^{-3 \theta \tau}\left(-6 e^{2 \theta \tau}(2 \theta \tau-5)-15 e^{\theta \tau}+e^{3 \theta \tau}(-6 \theta \tau(\theta \tau-3)-17)+2\right)}{6 \theta^{5}} .
$$

So, we finally arrive at the least singular solution

$$
\varphi_{2}=x \frac{h(\tau)}{\hat{t}(\tau)} G(x, \hat{t}(\tau))
$$

We note that in the limit $\theta \rightarrow 0$, this is consistent with the result (25) found earlier for $\varphi_{2}$ when $\theta=0$, since

$$
\frac{h(\tau)}{\hat{t}(\tau)}=-\frac{2}{5} \tau^{2}+\frac{7}{60} \theta \tau^{3}-\frac{229}{8400} \theta^{2} \tau^{4}+\frac{181}{33600} \theta^{3} \tau^{5}+\mathcal{O}\left(\theta^{4} \tau^{6}\right) .
$$

Although no approximation is needed at this stage, this and the previous series expansions are noted also because $0<\tau \leqslant 1$ by construction and, in practice, $\theta=(r-q) T$ is usually small. These power series representations are often actually more useful than the exact representations of the solutions. This is because the exact representations involve differences and, worse still ratios of differences, of functions which cancel up to relatively high powers of $\theta$ as $\theta$ becomes small, leading to considerable rounding error.

We also note that as $\zeta \rightarrow \pm \infty$, this term in the asymptotic expansion vanishes exponentially fast.

\subsubsection{Solution of third-order problem for $r \neq q$}

The PDE for $\varphi_{3}$ is in general given by

$$
\frac{\partial \varphi_{3}}{\partial \tau}-\eta^{*}(\tau)^{2} \frac{\partial^{2} \varphi_{3}}{\partial \zeta^{2}}+\theta \zeta \frac{\partial \varphi_{3}}{\partial \zeta}=2 \eta^{*}(\tau) \zeta \frac{\partial^{2} \varphi_{2}}{\partial \zeta^{2}}+\zeta^{2} \frac{\partial^{2} \varphi_{1}}{\partial \zeta^{2}}
$$

subject to the initial condition $\varphi_{3}(\zeta, 0)=0$. We already know that

$$
\zeta^{2} \frac{\partial^{2} \varphi_{1}}{\partial \zeta^{2}}=x^{2} \frac{\partial^{2} \varphi_{1}}{\partial x^{2}}=x^{2} G(x, \hat{t}(\tau))
$$

and we can deduce that

$$
\frac{\partial^{2} \varphi_{2}}{\partial \zeta^{2}}=e^{-2 \theta \tau} \frac{\partial^{2} \varphi_{2}}{\partial x^{2}}=e^{-2 \theta \tau} \frac{x\left(x^{2}-6 \hat{t}(\tau)\right)}{4 \hat{t}^{2}(\tau)} \frac{h(\tau)}{\hat{t}(\tau)} G(x, \hat{t}(\tau)) .
$$

The PDE for $\varphi_{3}$ can therefore be written as

$$
\frac{\partial \varphi_{3}}{\partial \tau}-\eta^{*}(\tau)^{2} \frac{\partial^{2} \varphi_{3}}{\partial \zeta^{2}}+\theta \zeta \frac{\partial \varphi_{3}}{\partial \zeta}=\left(x^{2}\left(1-\frac{3 h(\tau) \eta^{*}(\tau) e^{-\theta \tau}}{\hat{t}^{2}(\tau)}\right)+x^{4} \frac{h(\tau) \eta^{*}(\tau) e^{-\theta \tau}}{2 \hat{t}^{3}(\tau)}\right) G(x, \hat{t}(\tau)),
$$

and, regarding $\varphi_{3}$ as a function of the variables $x$ and $\hat{t}$, this becomes

$$
\frac{\partial \varphi_{3}}{\partial \hat{t}}-\frac{\partial^{2} \varphi_{3}}{\partial x^{2}}=\left(x^{2}\left(1-\frac{3 h(\tau) \eta^{*}(\tau) e^{-\theta \tau}}{\hat{t}^{2}(\tau)}\right)+x^{4} \frac{h(\tau) \eta^{*}(\tau) e^{-\theta \tau}}{2 \hat{t}^{3}(\tau)}\right) \frac{G(x, \hat{t})}{\left(\eta^{*}(\tau) e^{-\theta \tau}\right)^{2}} .
$$


As before, we make the assumption of a suitable solution, in the form of the product of a polynomial in $x$ with $\hat{t}$ dependent coefficients and $G$,

$$
\varphi_{3}=\left(f_{1}(\hat{t})+f_{2}(\hat{t}) x^{2}+f_{3}(\hat{t}) x^{4}\right) G(x, \hat{t}) .
$$

On differentiating and substituting into the PDE, we find that

$$
\begin{aligned}
\frac{\partial \varphi_{3}}{\partial \hat{t}}-\frac{\partial^{2} \varphi_{3}}{\partial x^{2}}= & \left(\left(\frac{d f_{3}}{d \hat{t}}+\frac{4 f_{3}(\hat{t})}{\hat{t}}\right) x^{4}+\left(\frac{d f_{2}}{d \hat{t}}+\frac{2 f_{2}(\hat{t})}{\hat{t}}-12 f_{3}(\hat{t})\right) x^{2}\right. \\
& \left.+\left(\frac{d f_{1}}{d \hat{t}}-2 f_{2}(\hat{t})\right)\right) G(x, \hat{t}(\tau)) .
\end{aligned}
$$

Matching powers of $x$ gives us three first-order ODEs for $f_{1}(\hat{t}), f_{2}(\hat{t})$ and $f_{3}(\hat{t})$.

As $\hat{t}(\tau)$ is defined by

$$
\frac{d \hat{t}(\tau)}{d \tau}=\eta^{*}(\tau)^{2} e^{-2 \theta \tau}, \quad \hat{t}(0)=0
$$

we can revert to $\tau$ as our independent time variable, introduce suitable integrating factors and show that these three ODES may be written as

$$
\begin{aligned}
\frac{d\left(\hat{t}(\tau)^{4} f_{3}(\tau)\right)}{d \tau} & =\frac{1}{2} \hat{t}(\tau) h(\tau) \eta^{*}(\tau) e^{-\theta \tau} \\
\frac{d\left(\hat{t}(\tau)^{2} f_{2}(\tau)\right)}{d \tau} & =\left(1+12 f_{3}(\tau) \eta^{*}(\tau)^{2} e^{-2 \theta \tau}\right) \hat{t}(\tau)^{2}-3 h(\tau) \eta^{*}(\tau) e^{-\theta \tau} \\
\frac{d f_{1}(\tau)}{d \tau} & =2 f_{2}(\tau) \eta^{*}(\tau)^{2} e^{-2 \theta \tau}
\end{aligned}
$$

subject to minimum singularity conditions at $\tau=0$. These may be integrated directly, in sequence.

First, for $f_{3}(\tau)$, we obtain

$$
f_{3}(\tau)=\frac{F(\tau)}{\hat{t}(\tau)^{4}}
$$

where

$$
\begin{aligned}
F(\tau) & =\frac{1}{2} \int_{0}^{\tau} h\left(\tau^{\prime}\right) \hat{t}\left(\tau^{\prime}\right) \eta^{*}\left(\tau^{\prime}\right) e^{-\theta \tau^{\prime}} d \tau^{\prime} \\
& =\frac{1}{4} \int_{0}^{\tau} h\left(\tau^{\prime}\right) \frac{d h\left(\tau^{\prime}\right)}{d \tau^{\prime}} d \tau^{\prime} \\
& =\frac{1}{8} h(\tau)^{2} .
\end{aligned}
$$


Expanding in powers of $\theta=(r-q) T$, we find that

$$
\begin{aligned}
f_{3}(\tau)= & \frac{h(\tau)^{2}}{8 \hat{t}(\tau)^{4}} \\
= & \frac{9}{50} \tau^{-2}+\frac{33}{200} \frac{\theta}{\tau}+\frac{6731}{112000} \theta^{2}+\frac{209}{22400} \tau \theta^{3}-\frac{57}{6272000} \tau^{2} \theta^{4}-\frac{2851}{18816000} \tau^{3} \theta^{5} \\
& +\mathcal{O}\left(\tau^{4} \theta^{6}\right),
\end{aligned}
$$

which is consistent with the formula found earlier for $\theta=0$.

For $f_{2}(\tau)$ and $f_{1}(\tau)$, direct integration leads to very complicated closed-form expressions with the property that both the numerator and denominator behave as high powers of $\theta$ as $\theta \rightarrow 0$. It is simpler and better to simply state, and use, the resulting series expansions for these functions, which are

$$
\begin{aligned}
f_{2}(\tau)= & -\frac{11}{175} \tau-\frac{87}{5600} \tau^{2} \theta-\frac{151}{504000} \tau^{3} \theta^{2}+\frac{71}{192000} \tau^{4} \theta^{3}-\frac{10849}{1552320000} \tau^{5} \theta^{4}-\frac{12311}{993484800} \tau^{6} \theta^{5} \\
& +\mathcal{O}\left(\tau^{7} \theta^{6}\right)
\end{aligned}
$$

and

$$
\begin{aligned}
f_{1}(\tau)= & -\frac{11}{350} \tau^{4}+\frac{53}{2800} \tau^{5} \theta-\frac{1543}{216000} \tau^{6} \theta^{2}+\frac{4217}{2016000} \tau^{7} \theta^{3}-\frac{4843981}{9313920000} \tau^{8} \theta^{4}+\frac{4295503}{37255680000} \tau^{9} \theta^{5} \\
& +\mathcal{O}\left(\tau^{10} \theta^{6}\right) .
\end{aligned}
$$

These expressions are consistent with the zero drift case (26).

Note again that as $\zeta \rightarrow \pm \infty, \varphi_{3}$ vanishes exponentially.

\subsection{Summary of the third-order solution for $r \neq q$}

It is convenient to gather together the following summary of the various functions for the inner solution when $r \neq q$. We first remind the reader of the basic transformations

$$
\begin{gathered}
\theta=(r-q) T, \quad \tau=\frac{T-t}{T}, \quad \epsilon^{2}=\frac{1}{2} \sigma^{2} T, \\
\eta=\frac{I-K T}{S T}, \quad V=S e^{-q(T-t)} \psi,
\end{gathered}
$$

the further changes of variable

$$
\begin{gathered}
\eta^{*}(\tau)=\frac{1}{\theta}\left(1-e^{\theta \tau}\right), \quad \eta=\eta^{*}(\tau)+\epsilon \zeta=\eta^{*}(\tau)+\epsilon e^{\theta \tau} x, \\
x=e^{-\theta \tau} \zeta=\frac{1}{\epsilon}\left(\eta-\eta^{*}(\tau)\right) e^{-\theta \tau}, \quad \hat{t}(\tau)=\frac{1}{2 \theta^{3}}\left(2 \theta \tau-3+4 e^{-\theta \tau}-e^{-2 \theta \tau}\right),
\end{gathered}
$$


the auxiliary functions defined by

$$
\begin{gathered}
G(x, \hat{t})=\frac{1}{\sqrt{4 \pi \hat{t}}} \exp \left(-\frac{x^{2}}{4 \hat{t}}\right), \\
h(\tau)=\frac{1}{6 \theta^{5}} e^{-3 \theta \tau}\left(2-15 e^{\theta \tau}-6(2 \theta \tau-5) e^{2 \theta \tau}+(-6 \theta \tau(\theta \tau-3)-17) e^{3 \theta \tau}\right),
\end{gathered}
$$

and the asymptotic expansion

$$
\psi=\epsilon \varphi_{1}+\epsilon^{2} \varphi_{2}+\epsilon^{3} \varphi_{3}+\cdots
$$

The first-, second- and third-order terms in this asymptotic expansion are given by

$$
\begin{aligned}
& \varphi_{1}=x \mathrm{~N}\left(\frac{x}{\sqrt{2 \hat{t}(\tau)}}\right)+\sqrt{\frac{\hat{t}(\tau)}{\pi}} \exp \left(-\frac{x^{2}}{4 \hat{t}(\tau)}\right) \\
& \varphi_{2}=\frac{h(\tau)}{\hat{t}(\tau)} x G(x, \hat{t}(\tau)) \\
& \varphi_{3}=\left(f_{1}(\tau)+f_{2}(\tau) x^{2}+f_{3}(\tau) x^{4}\right) G(x, \hat{t}(\tau)),
\end{aligned}
$$

where the functions $f_{3}, f_{2}$ and $f_{1}$ are given by the series expansions

$$
\begin{aligned}
f_{3}(\tau)= & \frac{h(\tau)^{2}}{8 \hat{t}(\tau)^{4}} \\
= & \frac{9}{50} \tau^{2}+\frac{33}{200} \theta \tau+\frac{6731}{112000} \theta^{2}+\frac{209}{22400} \tau \theta^{3}-\frac{57}{6272000} \tau^{2} \theta^{4}-\frac{2851}{18816000} \tau^{3} \theta^{5} \\
& +\mathcal{O}\left(\tau^{4} \theta^{6}\right), \\
f_{2}(\tau)= & -\frac{11}{175} \tau-\frac{87}{5600} \tau^{2} \theta-\frac{151}{504000} \tau^{3} \theta^{2}+\frac{71}{192000} \tau^{4} \theta^{3}-\frac{10849}{1552320000} \tau^{5} \theta^{4}-\frac{12311}{993484800} \tau^{6} \theta^{5} \\
& +\mathcal{O}\left(\tau^{7} \theta^{6}\right), \\
f_{1}(\tau)= & -\frac{11}{350} \tau^{4}+\frac{53}{2800} \tau^{5} \theta-\frac{1543}{216000} \tau^{6} \theta^{2}+\frac{4217}{2016000} \tau^{7} \theta^{3}-\frac{4843981}{9313920000} \tau^{8} \theta^{4}+\frac{4295503}{37255680000} \tau^{9} \theta^{5} \\
& +\mathcal{O}\left(\tau^{10} \theta^{6}\right) .
\end{aligned}
$$

Hence, we may determine the contract value to third order in $\epsilon=\sigma \sqrt{T / 2}$. It is perhaps helpful to take a step back from the details and observe what has been achieved. We have been able to determine the contract value as a series in the volatility using a combination of some changes of variables, one reference to the normal distribution, some polynomials and some exponential functions. It is now trivial to code this up in any computational environment, and work out the results. This is in effect an analogue of the Black-Scholes formula, at least in terms of its complexity, for an Asian rate option. We shall shortly make a comparison of the results with the known exact solution expressed as a contour 
integral. Before doing so, we shall introduce another low-volatility approximation based on the SDE. This will help us evaluate the quality of the asymptotic series developed above when the contour integral is difficult to evaluate, i.e. when $\epsilon$ is very small.

\section{Asymptotic solution of the SDE}

As a further check on our methodology, we now develop an alternative perturbation solution method based directly on the corresponding (risk-neutral) SDE, (4). For continuous sampling over the life of the option, this becomes

$$
d \eta_{t}=\left(\frac{1}{T}-(r-q) \eta_{t}\right) d t+\sigma \eta_{t} d W_{t}
$$

where $W_{t}$ is a standard Brownian motion. We also suppose that at $t=0$, we are given $\eta_{0}$. To treat this in the low-volatility limit, we let

$$
\eta_{t}=\eta_{t}^{0}+\sigma \eta_{t}^{1}
$$

The SDE for the first term, $\eta_{t}^{0}$, is the deterministic ODE

$$
d \eta_{t}^{0}=\left(\frac{1}{T}-(r-q) \eta_{t}^{0}\right) d t
$$

with the elementary solution

$$
\eta_{t}^{0}= \begin{cases}\eta_{0} e^{-(r-q) t}+\frac{1-e^{-(r-q) t}}{(r-q) T} & \text { if } r \neq q, \\ \eta_{0}+\frac{t}{T} & \text { if } r=q .\end{cases}
$$

The exact SDE for the second term is the SDE

$$
d \eta_{t}^{1}=-(r-q) \eta_{t}^{1} d t+\left(\eta_{t}^{0}+\sigma \eta_{t}^{1}\right) d W_{t}
$$

subject to the initial condition $\eta_{0}^{1}=0$. This SDE can be simplified in the usual way by setting

$$
\tilde{\eta}_{t}^{1}=e^{(r-q) t} \eta_{t}^{1}
$$

and substituting for the known form of $\eta_{t}^{0}$. For $r \neq q$, we obtain ${ }^{9}$

$$
d \tilde{\eta}_{t}^{1}=\left(\eta_{0}^{0}+\frac{e^{(r-q) t}-1}{(r-q) T}+\sigma \tilde{\eta}_{t}^{1}\right) d W_{t}
$$

which has the formal solution

$$
\tilde{\eta}_{t}^{1}=A_{t}+\sigma B_{t}+\mathcal{O}\left(\sigma^{2}\right)
$$

9 The results for $r=q$ follow by taking the limit $r \rightarrow q$ in the obvious manner. 
where

$$
\begin{aligned}
A_{t} & =\int_{0}^{t} d W_{s}\left(\eta_{0}^{0}+\frac{e^{(r-q) s}-1}{(r-q) T}\right), \\
B_{t} & =\int_{0}^{t} d W_{s} \int_{0}^{s} d W_{u}\left(\eta_{0}^{0}+\frac{e^{(r-q) u}-1}{(r-q) T}\right) .
\end{aligned}
$$

It is clear that $A_{t}^{1}$ is normally distributed with zero mean and variance $\Sigma^{2}$, given by

$$
\Sigma^{2}=\int_{0}^{t} d s\left(\eta_{0}^{0}+\frac{e^{(r-q) s}-1}{(r-q) T}\right)^{2} .
$$

If we recall that

$$
\eta_{t}=\eta_{t}^{0}+\sigma\left(A_{t}+\sigma B_{t}+\mathcal{O}\left(\sigma^{2}\right)\right) e^{-(r-q) t}
$$

it follows that if we neglect terms quadratic and higher order in $\sigma, \eta_{t}$ is normally distributed with mean $\eta_{t}^{0}$ and variance $e^{-2(r-q) t} \sigma^{2} \Sigma^{2}$.

We can now write down the value of $\psi$ based on the Feynman-Kac formula, to this order in $\sigma$. Elementary calculations show that

$$
\psi_{t}=\eta_{t}^{0} \mathrm{~N}_{c}\left(-\frac{\eta_{t}^{0} e^{(r-q)(T-t)}}{\sigma \Sigma}\right)-e^{-(r-q)(T-t)} \sigma \Sigma \mathrm{N}_{c}^{\prime}\left(\frac{\eta_{t}^{0} e^{(r-q)(T-t)}}{\sigma \Sigma}\right),
$$

where $\mathrm{N}_{c}$ denotes the complementary cumulative density for a standard normal distribution.

\section{Comparison of results}

In this section, we define an extension of a part of the useful family of test problems introduced by Geman and Eydeland and analysed for several methods by Fu, Madan and Wang (abbreviated henceforth to FMW) [2]. First we consider the seven cases as numbered by FMW. These all have zero dividend yield and positive $r$. The other parameters are all given as follows.

\begin{tabular}{ccclll}
\hline FMW No. & $S$ & $K$ & \multicolumn{1}{c}{$r$} & \multicolumn{1}{c}{$\sigma$} & $T$ \\
\hline 1 & 1.9 & 2 & 0.05 & 0.5 & 1 \\
2 & 2.0 & 2 & 0.05 & 0.5 & 1 \\
3 & 2.1 & 2 & 0.05 & 0.5 & 1 \\
4 & 2.0 & 2 & 0.02 & 0.1 & 1 \\
5 & 2.0 & 2 & 0.18 & 0.3 & 1 \\
6 & 2.0 & 2 & 0.0125 & 0.25 & 2 \\
7 & 2.0 & 2 & 0.05 & 0.5 & 2 \\
\hline
\end{tabular}


The results are as follows for these six cases. The results cited from Vecer are those in his RISK article [20] to six decimal places, noted as already agreeing with the results of $[12,14]$. The Linetsky data are high-precision data supplied by V. Linetsky and based on his work in [12]. The FMW-MC100 data are Monte Carlo data for the 'MC100' analysis from the paper by FMW [2]. The GYShaw results are those from the GY model run with the Shaw's code in [14] with a longer contour. Note that the cases are numbered differently by FMW and Linetsky, hence the first two columns ' $F$ ' and ' $L$ ' label their case numbers for ease of comparison. The results labelled 'MAE2' and 'MAE3' are the results from the asymptotics as far as $\varphi_{2}$ and $\varphi_{3}$, respectively and the results labelled 'FD' are from our implementation of Zhang's finite-difference method, described in Appendix B.

\begin{tabular}{cccccccccc}
\hline F & L & GYShaw & LVSDE & MAE2 & MAE3 & Linetsky & Vecer & FMW & FD \\
\hline 1 & 4 & 0.1931737903 & 0.1996 & 0.194472 & 0.193188 & 0.1931737903 & 0.193174 & 0.196 & 0.193174 \\
2 & 5 & 0.2464156905 & 0.2428 & 0.247732 & 0.246382 & 0.2464156905 & 0.246416 & 0.249 & 0.246416 \\
3 & 6 & 0.3062203648 & 0.2935 & 0.307604 & 0.306139 & 0.3062203648 & 0.306220 & 0.309 & 0.306220 \\
4 & 1 & 0.0559860415 & 0.0556 & 0.055997 & 0.055986 & 0.0559860415 & 0.055986 & 0.0565 & 0.055986 \\
5 & 2 & 0.2183875466 & 0.2104 & 0.218663 & 0.218369 & 0.2183875466 & 0.218388 & 0.220 & 0.218388 \\
6 & 3 & 0.1722687410 & 0.1710 & 0.172741 & 0.172263 & 0.1722687410 & 0.172269 & 0.172 & 0.172269 \\
7 & 7 & 0.3500952190 & 0.3404 & 0.353704 & 0.349909 & 0.3500952190 & 0.350095 & 0.348 & 0.350095 \\
\hline
\end{tabular}

We note that three of the approaches based on very different non-asymptotic methods agree to many significant figures, so there is now little doubt as to the correct answer to these problems. The two methods for which more details are given agree to 10 decimal places. The low-volatility SDE column gives an idea of the accuracy of the method in cases where one would not necessarily expect it to be very accurate, but it does moderately well. The important observation, however, is the accuracy of the third-order asymptotic results, which typically give four significant figure accuracy.

\subsection{Low-volatility test problems}

In order to probe the difficult case of low volatility, we now introduce some variants of FMW Case 4 as follows. The 'Low-vol S' column corresponds to the stochastic approximation introduced in Section 4. The FD and GYShaw methods are as before, with GYS-Mellin denoting the use of an asymptotic expansion to treat the low-volatility case via a Mellin transform method, as discussed in [18]. Note that the MAE3 results appear now to be essentially exact as far as can be tested.

\begin{tabular}{ccccll}
\hline Case & $S$ & $K$ & $r$ & \multicolumn{1}{c}{$\sigma$} & $T$ \\
\hline 4 & 2.0 & 2 & 0.02 & 0.1 & 1 \\
4A & 2.0 & 2 & 0.02 & 0.05 & 1 \\
4B & 2.0 & 2 & 0.02 & 0.01 & 1 \\
4C & 2.0 & 2 & 0.02 & 0.005 & 1 \\
4D & 2.0 & 2 & 0.02 & 0.001 & 1 \\
\hline
\end{tabular}


The results are as follows.

\begin{tabular}{clccccc}
\hline Case & GYShaw & GYS-Mellin & Low-vol SDE & MAE2 & MAE3 & FD \\
\hline 4 & 0.0559860 & 0.0559853 & 0.0555956 & 0.0559970 & 0.0559860 & 0.0559860 \\
4A & 0.0339412 & 0.0339485 & 0.0337509 & 0.0339426 & 0.0339412 & 0.0339412 \\
4B & NA & 0.0199383 & 0.0199188 & 0.0199277 & 0.0199278 & 0.0199278 \\
4C & NA & unstable & 0.0197357 & 0.0197357 & 0.0197357 & 0.0197357 \\
4D & NA & 0.0197353 & 0.0197353 & 0.0197353 & 0.0197353 & 0.0197353
\end{tabular}

\subsection{Test problems for $q>r$}

We now define analogues of the FMW test problems for the case where $q>r$. The simplest approach is to take the original set and but also to set $q=2 r$, so that we exactly reverse the risk-neutral drift. The parameters are shown below, using an asterisk to denote the reflected drift in the case numbers.

\begin{tabular}{cccllll}
\hline FMW No. & $S$ & $K$ & \multicolumn{1}{c}{$r$} & \multicolumn{1}{c}{$q$} & \multicolumn{1}{c}{$\sigma$} & $T$ \\
\hline $1^{*}$ & 1.9 & 2 & 0.05 & 0.1 & 0.5 & 1 \\
$2^{*}$ & 2.0 & 2 & 0.05 & 0.1 & 0.5 & 1 \\
$3^{*}$ & 2.1 & 2 & 0.05 & 0.1 & 0.5 & 1 \\
$4^{*}$ & 2.0 & 2 & 0.02 & 0.04 & 0.1 & 1 \\
$5^{*}$ & 2.0 & 2 & 0.18 & 0.36 & 0.3 & 1 \\
$6^{*}$ & 2.0 & 2 & 0.0125 & 0.025 & 0.25 & 2 \\
$7^{*}$ & 2.0 & 2 & 0.05 & 0.1 & 0.5 & 2 \\
\hline
\end{tabular}

The results are as follows. In this case, given that there is not a published set of high-precision values, we confine attention to six significant figures.

\begin{tabular}{cllll}
\hline FMW No. & GYShaw & \multicolumn{1}{c}{ MAE2 } & MAE3 & \multicolumn{1}{c}{ FD } \\
\hline $1^{*}$ & 0.147562 & 0.148852 & 0.147618 & 0.147562 \\
$2^{*}$ & 0.191747 & 0.193010 & 0.191760 & 0.191747 \\
$3^{*}$ & 0.242316 & 0.243595 & 0.242283 & 0.242316 \\
$4^{*}$ & 0.0357853 & 0.0357961 & 0.0357854 & 0.0357853 \\
$5^{*}$ & 0.0522607 & 0.0524892 & 0.0522755 & 0.0522607 \\
$6^{*}$ & 0.145308 & 0.145771 & 0.145310 & 0.145308 \\
$7^{*}$ & 0.240495 & 0.243819 & 0.240564 & 0.240495 \\
\hline
\end{tabular}

\subsubsection{Low volatility and $q>r$ tests}

To see what happens for low volatilities, we consider the test problems used previously with the volatility tending to zero and also set $q=2 r$. The parameters are therefore as follows. 


\begin{tabular}{lcccccc}
\hline Case & $S$ & $K$ & $r$ & $q$ & \multicolumn{1}{c}{$\sigma$} & $T$ \\
\hline $4^{*}$ & 2.0 & 2 & 0.02 & 0.04 & 0.1 & 1 \\
$4 \mathrm{~A}^{*}$ & 2.0 & 2 & 0.02 & 0.04 & 0.05 & 1 \\
4B $^{*}$ & 2.0 & 2 & 0.02 & 0.04 & 0.01 & 1 \\
4C* $^{*}$ & 2.0 & 2 & 0.02 & 0.04 & 0.005 & 1 \\
4D* & 2.0 & 2 & 0.02 & 0.04 & 0.001 & 1 \\
\hline
\end{tabular}

The corresponding results are as follows.

\begin{tabular}{lcllcc}
\hline Case & GYS-full & Low-vol SDE & \multicolumn{1}{c}{ MAE2 } & MAE3 & FD \\
\hline $4^{*}$ & 0.0357853 & 0.0361954 & 0.0357961 & 0.0357854 & 0.0357853 \\
$4 A^{*}$ & 0.0140247 & 0.0142171 & 0.0140261 & 0.0140248 & 0.0140247 \\
$4 B^{*}$ & NA & 0.000199435 & 0.000190211 & 0.000190254 & 0.000190254 \\
$4 C^{*}$ & NA & $4.33117 \times 10^{-7}$ & $3.78827 \times 10^{-7}$ & $3.7991 \times 10^{-7}$ & $3.7993 \times 10^{-7}$ \\
4D* & NA & $\mathcal{O}\left(10^{-68}\right)$ & $\mathcal{O}\left(10^{-70}\right)$ & $\mathcal{O}\left(10^{-70}\right)$ & $\mathcal{O}\left(10^{-72}\right)$ \\
\hline
\end{tabular}

\subsection{Test problems for $q=r$}

This case is a particularly stringent one as we have up to five orders of perturbation theory at our disposal and we can compare with a detailed contour integral solution expressed in terms of Bessel functions. We now define analogues of the FMW test problems for the case where $r=q$. The simplest thing to do is to take the original set and but also to set $q=r$, so that we zero the risk-neutral drift of $S$. The parameters are shown below, using a double asterisk to denote the zeroed drift in the case numbers.

\begin{tabular}{cccllll}
\hline FMW No. & $S$ & $K$ & \multicolumn{1}{c}{$r$} & \multicolumn{1}{c}{$q$} & \multicolumn{1}{c}{$\sigma$} & $T$ \\
\hline $1^{* *}$ & 1.9 & 2 & 0.05 & 0.05 & 0.5 & 1 \\
$2^{* *}$ & 2.0 & 2 & 0.05 & 0.05 & 0.5 & 1 \\
$3^{* *}$ & 2.1 & 2 & 0.05 & 0.05 & 0.5 & 1 \\
$4^{* *}$ & 2.0 & 2 & 0.02 & 0.02 & 0.1 & 1 \\
$5^{* *}$ & 2.0 & 2 & 0.18 & 0.18 & 0.3 & 1 \\
$6 * *$ & 2.0 & 2 & 0.0125 & 0.0125 & 0.25 & 2 \\
$7 * *$ & 2.0 & 2 & 0.05 & 0.05 & 0.5 & 2
\end{tabular}

The results are as follows. We confine attention to six significant figures. These exact results are labelled 'CIBess' (contour integral of the Bessel function form of the hypergeometric function that is given in (A 1) in the appendix). 


\begin{tabular}{cllllll}
\hline FMW No. & CIBess & MAE2 & MAE3 & MAE4 & MAE5 & \multicolumn{1}{c}{ FD } \\
\hline $1^{* *}$ & 0.169202 & 0.170494 & 0.169238 & 0.169192 & 0.169201 & 0.169202 \\
$2^{* *}$ & 0.217815 & 0.219096 & 0.217805 & 0.217805 & 0.217815 & 0.217815 \\
$3^{* *}$ & 0.272924 & 0.274251 & 0.272869 & 0.272914 & 0.272925 & 0.272924 \\
$4 * *$ & 0.0451431 & 0.0451537 & 0.0451431 & 0.0451431 & 0.0451431 & 0.0451431 \\
$5^{* *}$ & 0.115188 & 0.115432 & 0.115188 & 0.115188 & 0.115188 & 0.115188 \\
$6^{* *}$ & 0.158380 & 0.158846 & 0.158378 & 0.158378 & 0.158380 & 0.158380 \\
$7 * *$ & 0.291315 & 0.294737 & 0.291264 & 0.291264 & 0.291316 & 0.291315 \\
\hline
\end{tabular}

It should be noted that the MAE3 results are all correct to at least four significant figures or better, and the MAE5 results are exact for all practical purposes.

\subsubsection{Low volatility and $q=r$ tests}

To see what happens for low volatilities, we consider the test problems used previously with the volatility tending to zero and also set $q=r$. The parameters are therefore as follows.

\begin{tabular}{lcccccc}
\hline Case & $S$ & $K$ & $r$ & $q$ & $\sigma$ & $T$ \\
\hline $4 * *$ & 2.0 & 2 & 0.02 & 0.02 & 0.1 & 1 \\
$4 \mathrm{~A}^{* *}$ & 2.0 & 2 & 0.02 & 0.02 & 0.05 & 1 \\
$4 \mathrm{~B}^{* *}$ & 2.0 & 2 & 0.02 & 0.02 & 0.01 & 1 \\
$4 \mathrm{C}^{* *}$ & 2.0 & 2 & 0.02 & 0.02 & 0.005 & 1 \\
$4 \mathrm{D} * *$ & 2.0 & 2 & 0.02 & 0.02 & 0.001 & 1 \\
\hline
\end{tabular}

The corresponding results are as follows.

\begin{tabular}{lcllll}
\hline Case & CIBess & Low-vol SDE & \multicolumn{1}{c}{ MAE2 } & \multicolumn{1}{c}{ MAE3 } & \multicolumn{1}{c}{ FD } \\
\hline $4^{* *}$ & 0.0451431 & 0.0451537 & 0.0451537 & 0.0451431 & 0.0451431 \\
4A $^{* *}$ & 0.0225755 & 0.0225769 & 0.0225769 & 0.0225755 & 0.0225755 \\
4B $^{* *}$ & NA & 0.00451537 & 0.00451537 & 0.00451536 & 0.00451536 \\
4 C $^{* *}$ & NA & 0.00225769 & 0.00225769 & 0.00225768 & 0.00225768 \\
$4 D^{* *}$ & NA & 0.000451537 & 0.000451537 & 0.000451537 & 0.000451537 \\
\hline
\end{tabular}

We note that in these cases, given that $\zeta=0$ for all cases where $S=K$ and $r=q$, the MAE4 results are the same as MAE3. Where we know the exact result, we see that the MAE3 values are good to at least six significant figures. Going to MAE5 makes no difference to the results with the given precision. 


\section{Conclusion}

We have developed the partial differential equation approach to Asian options in such a way as to allow both an easy derivation of the exact solution for the rate call as a Laplace transform and as a power series in

$$
\epsilon=\sigma \sqrt{\frac{T}{2}}
$$

When carried out to third order, the expansion approach agrees with the Laplace transform method to typically four significant figures for real-world volatilities and a range of values of $(r-q) T$. The expansion method is essentially exact as $\epsilon \rightarrow 0$. When $r=q$, we have a very accurate fifth-order expansion which gives excellent agreement with the contour integral solution optimised for this case. The expansion approach gives a formula that is little more complicated than the original Black-Scholes model for European options and may be readily implemented in any computational environment. An online Mathematica notebook demonstrating some of these calculations is available [17]. These models may be used stand-alone or for checking numerical implementations of e.g. the Vecer model. Our model is essentially an accurate analytical solution of Vecer's PDE.

The methodology developed here may in principle be applied to many other financial problems of interest. Problems that immediately come to mind are the extension of the present to work to include

- the rate call with discrete sampling;

- the inclusion of discrete dividends;

- the strike call.

Within the PDE framework, it is very clear how this methodology can be iterated through higher powers of $\epsilon$. Indeed, we have demonstrated this very explicitly to high order in the case of zero risk-neutral drift: $r=q$. It would be interesting to consider how precisely this would take place within the SDE framework. While the SDE can be formally iterated in powers of $\sigma$, the question arises as to how one could get explicit formulae for expectations within such an expansion?

\section{References}

[1] Abramowitz, M. \& Stegun, I. A. (1970) Handbook of Mathematical Functions, Dover Edition, New York.

[2] Fu, M., Madan, D. \& Wang, T. (1998) Pricing continuous time Asian options: A comparison of Monte Carlo and Laplace transform inversion methods. J. Comp. Fin. 2, 49-74.

[3] Geman, H. \& Eydeland, A. (1995) Domino effect: Inverting the Laplace transform. RISK Magazine, March 1995.

[4] Geman, H. \& Yor, M. (1993) Bessel processes, Asian options and perpetuities. Math. Finance 3, 349-375.

[5] Gradshteyn, I. S. \& Ryzhik, I. M. (1980) Table of Integrals, Series and Products, Corrected and enlarged edition, Academic Press, New York.

[6] Hinch, E. J. (1991) Perturbation Methods, Cambridge University Press, Cambridge.

[7] Howison, S. D. (2005) Matched asymptotic expansions in financial engineering. J. Engrg. Math. 53, 385-406. 
[8] Howison, S. D. (2007) A matched asymptotic expansions approach to continuity corrections for discretely sampled options. Part 2: Bermudan options. Appl. Math. Finance 14, 91-104.

[9] Howison, S. D. \& Steinberg, M. (2007) A matched asymptotic expansions approach to continuity corrections for discretely sampled options. Part 1: Barrier options. Appl. Math. Finance 14, 63-89.

[10] Ingersoll, J. E. (1987) Theory of Financial Decision Making, Rowman \& Littlefield Studies in Financial Economics, Rowman and Littlefield, Savage, Maryland, USA.

[11] LewIS, A. (2002) Asian connections. In: Algorithms, Wilmott Magazine, September 2002, pp. 5763.

[12] Linetsky, V. (2004) Spectral expansions for Asian (average price) options. Oper. Res. 52, 856.

[13] Rogers, L .C. G. \& SHI, Z. (1995) The value of an Asian option. J. Appl. Probab. 32, 1077-1088.

[14] Shaw, W. T. (1998) Modelling Financial Derivatives with Mathematica, Cambridge University Press, Cambridge, UK.

[15] Shaw, W. T. (1998) A Reply to "Pricing continuous Asian options: A comparison of Monte Carlo and Laplace transform inversion methods" by $\mathrm{Fu}$, Madan and Wang. J. Comp. Fin. 2, 49-74. Working Paper, 2000. URL: http://www.mth.kcl.ac.uk/ shaww/ web_page/papers/JCFReply.pdf, http://www.mth.kcl.ac.uk/ shaww/web_page/papers/JCFReply.nb.

[16] Shaw, W. T. Transform Calculus, Conformal Mapping and Applications to Mathematical Finance, OCIAM, Oxford (2003) and Judge Business School (2004) presentations (seminar working paper based on work with A. MacDonald and J. Dewynne). URL: http:// www.mth.kcl.ac.uk/ shaww/web_page/talks/conformal.pdf.

[17] SHaw, W. T. Mathematica implementations of Asian option valuation by contour integration, Mathematica Note Book (2007 version). URL: http://www.mth.kcl.ac.uk/ 〜shaww/web_page/papers/asianmathematica.nb.

[18] Shaw, W. T. (2003) Pricing Asian Options by Contour Integration, Including Asymptotic Methods for Low Volatility, Working Paper, Oxford Mathematical Institute. URL: http://www.mth.kcl.ac.uk/ shaww/web_page/papers/lowvol.pdf, http://www.mth. kcl.ac.uk/ shaww/web_page/papers/lowvol.nb.

[19] Van Dyke, M. (1978) Perturbation Methods in Fluid Mechanics, Parabolic Press, Stanford, CA.

[20] Vecer, J. (2001) A new PDE approach for pricing arithmetic Asian options. J. Comp. Fin. 4, $105-113$.

[21] VeCER, J. (2002) Unified Asian pricing. RISK 15(6), 113-116.

[22] Wilmott, P., Dewynne, J. \& Howison, S. (1993) Option Pricing, Mathematical Models and Computation, Oxford Financial Press, Oxford.

[23] Wilmott, P., Howison, S. \& Dewynne, J. (1995) Mathematics of Financial Derivatives, Cambridge University Press, Cambridge.

[24] Wong, E. (1964) The construction of a class of stationary Markoff processes. In: Proceedings of Symposia in Applied Mathematics, Vol. XVI, Stochastic Processes in Mathematical Physics and Engineering, American Mathematical Society, Providence, RI, pp. 265-276.

[25] Yor, M. (2001) Exponential Functionals of Brownian Motion and Related Processes, Springer Finance, Berlin.

[26] Zhang, J. E. (2001) A semi-analytical method for pricing and hedging continuously sampled arithmetic average rate options. J. Comp. Fin. 5, 59-79.

[27] Zhang, J. E. (2003) Pricing continuously sampled Asian options with perturbation method. J. Futures Mkts 23(6), 535-560.

\section{Appendix A \\ Reduction of the hyper-geometric contour integral to Bessel form for special values of $r-q$}

The Laplace transform solution of equation (11) can be represented in a number of equivalent ways. It is in fact useful to recast the transform back as an integral, though 
not in the form given by GY. Three representations are interesting. These representations will not be justified here - they can be deduced from various identities and results in Gradshteyn and Ryzik [5] and Abramowitz and Stegun [1]. We can write, using some well known integral identities,

$$
\hat{C}(\alpha, p)=\frac{\Gamma\left(\frac{1}{2}(\mu+v+4)\right)}{\Gamma\left(\frac{1}{2}(\mu-v-2)\right)} \frac{2^{v+3}}{p(p-2 v-2)} \int_{0}^{\infty} \frac{1}{x^{v+3}} J_{\mu}(x) e^{-\alpha x^{2} / 2} d x .
$$

Applying a Kummer transformation and another identity leads to the simpler form

$$
\hat{C}(\alpha, p)=\frac{\alpha^{v+3} e^{-\frac{1}{2 \alpha}}}{p(p-2 v-2)} \int_{0}^{\infty} x^{v+3} I_{\mu}(x) e^{-\alpha x^{2} / 2} d x .
$$

Finally, we note our third representation, in terms of a Mellin transform

$$
\hat{C}(\alpha, p)=\frac{1}{2 \pi i} \frac{1}{p(p-2 v-2)} \frac{\Gamma(b)}{\Gamma(a)} \int_{-i \infty}^{i \infty}(2 \alpha)^{-z} \frac{\Gamma(z) \Gamma(a-z)}{\Gamma(b+z)} d z,
$$

where $a=\mu / 2-v / 2-1, b=\mu / 2+v / 2+2$. Any of these representations make it clear that there is a collapse in complexity when $v=-3$ ! This might seem like an odd candidate for a special case - it does in fact correspond to zero drift in the variable $u=1 / \eta$. In this case, we can carry out the integration in both the first two representations to obtain a simpler representation

$$
\hat{C}(\alpha, p)=\frac{e^{\frac{-1}{4 \alpha}}}{p(p+4)} \sqrt{\frac{\pi}{2 \alpha}} I_{\frac{\mu}{2}}\left(\frac{1}{4 \alpha}\right), \quad \mu=\sqrt{2 p+9} .
$$

There are also simplifications when $n u=-2-1,0,1,2,3,4 \ldots$, when the hyper-geometric function can be reduced to a finite sum of Bessel functions. Only the first few of these will be noted here. Note that $v=-1$ is particularly important as it corresponds to the situation $r=q$. All the noted integer cases for $v$ can be derived from that above for $v=-3$ and the following that is valid for $v=-2$ :

$$
\hat{C}(\alpha, p)=\frac{e^{\frac{-1}{4 \alpha}}}{p(p+2)} \sqrt{\frac{\pi}{2 \alpha}} \frac{1}{2}\left(I_{\frac{\mu}{2}-\frac{1}{2}}\left(\frac{1}{4 \alpha}\right)+I_{\frac{\mu}{2}+\frac{1}{2}}\left(\frac{1}{4 \alpha}\right)\right), \quad \mu=\sqrt{2 p+4}
$$

In general, for the sequence $v=2 k-3, k=0,1,2, \ldots$,

$$
\hat{C}(\alpha, p)=2^{k} \alpha^{2 k} \frac{e^{-\frac{1}{2 \alpha}}}{p(p+4-4 k)}\left(-\frac{d}{d \alpha}\right)^{k}\left(e^{\frac{1}{4 \alpha}} \sqrt{\frac{\pi}{2 \alpha}} I_{\frac{\mu}{2}}\left(\frac{1}{4 \alpha}\right)\right) .
$$

Applying this result with $k=1$, we obtain an important result for $v=-1, r=q$, which after the use of some Bessel identities reduces to

$$
\hat{C}(\alpha, p)=\frac{e^{\frac{-1}{4 \alpha}}}{p^{2}} \sqrt{\frac{\pi}{8 \alpha}}\left((1+2 \alpha(\mu+1)) I_{\frac{\mu}{2}}\left(\frac{1}{4 \alpha}\right)+I_{\frac{\mu}{2}+1}\left(\frac{1}{4 \alpha}\right)\right), \quad \mu=\sqrt{2 p+1} .
$$


For the even sequence $v=2 k-2, k=0,1,2, \ldots$, we obtain the analogous result

$$
\hat{C}(\alpha, p)=2^{k} \alpha^{2 k+1} \frac{e^{-\frac{1}{2 \alpha}}}{p(p+2-4 k)}\left(-\frac{d}{d \alpha}\right)^{k}\left\{e^{\frac{1}{4 \alpha}} \sqrt{\frac{\pi}{2 \alpha}} \frac{1}{2 \alpha}\left(I_{\frac{\mu-1}{2}}\left(\frac{1}{4 \alpha}\right)+I_{\frac{\mu+1}{2}}\left(\frac{1}{4 \alpha}\right)\right)\right) .
$$

What is interesting about these expressions is that they reveal the essential problem of inversion - we need to understand the Laplace inverse of a Bessel function whose index is the (square root of the) Laplace transform variable. These expressions allow a formal analytical inverse to be extracted for such special cases in terms of Kontorovich-Lebedev transforms. We will not discuss the analytics of this here as our use of these Bessel representations is merely to facilitate the numerical computation of the contour integral when $r=q$. This is carried out using numerical inversion of the representation (A 1). Such integrations proceed up to an order of magnitude faster than direct use of the original hyper-geometric function, when carried out in Mathematica.

\section{Appendix B}

\section{Finite-difference method}

Following Zhang [26], we use the transformations

$$
\begin{gathered}
\xi=e^{q(T-t)} \frac{K T-I}{S}-\frac{1-e^{-(r-q)(T-t)}}{r-q}, \quad \tau=T-t, \\
V(S, I, t)=\frac{S}{T} e^{-q(T-t)} f(\xi, \tau)
\end{gathered}
$$

to reduce the problem for an average-strike call to

$$
\frac{\partial f}{\partial \tau}=\frac{1}{2} \sigma^{2}(\xi+b(\tau))^{2} \frac{\partial^{2} f}{\partial \xi^{2}}, \quad f(\xi, 0)=\max (-\xi, 0)
$$

where

$$
b(\tau)=\frac{1}{r-q}\left(1-e^{-(r-q)(T-t)}\right) .
$$

If $r=q$, we use the obvious limit and for $|r-q| \ll 1$, we use the first 10 terms of the Taylor expansion of this expression.

As in Zhang [26], we write

$$
f(\xi, \tau)=f_{0}(\xi, \tau)+f_{1}(\xi, \tau)
$$

where

$$
\frac{\partial f_{0}}{\partial \tau}=b(\tau)^{2} \frac{\partial^{2} f_{0}}{\partial \xi^{2}}, \quad f_{0}(\xi, 0)=\max (-\xi, 0)
$$

Following the same line of reasoning as in the body of the paper, we find that

$$
f_{0}(\xi, \tau)=-\xi \mathrm{N}\left(\frac{-\xi}{\sqrt{2 \eta}}\right)+\sqrt{\frac{\eta}{\pi}} e^{-\xi^{2} / 4 \eta}
$$


where

$$
\eta(\tau)=\frac{1}{2} \sigma^{2} \int_{0}^{\tau} b(w)^{2} d w
$$

The problem for $f_{1}$ is then

$$
\frac{\partial f_{1}}{\partial \tau}-\frac{1}{2} \sigma^{2}(\xi+b(\tau))^{2} \frac{\partial^{2} f_{1}}{\partial \xi^{2}}=\xi(\xi+2 b(\tau)) \frac{\partial^{2} f_{0}}{\partial \xi^{2}}, \quad f_{1}(\xi, 0)=0 .
$$

We solve for $f_{1}(\xi, \tau)$ using the finite-difference procedure described in Zhang [26], with zero function value conditions at the ends of the $\xi$-grid, and Zhang's numerical parameters. We were able to reproduce his result that, with his numerical parameters, zero-gradient and zero-curvature boundary conditions at the ends of the $\xi$-grid made no effective difference to the numerical solution. We also solved the problem for $f$ directly using a CrankNicolson scheme, with zero-curvature boundary conditions, and found the maximum and root-mean-square differences between these solutions and those from Zhang's method were $\mathcal{O}\left(10^{-8}\right)$ over the range of financial parameters of interest and again using Zhang's numerical parameters.

Finally, note that where it is necessary to interpolate prices, we do so using $f_{0}$ and the Matlab spline function with the numerical values of $f_{1}$ on its finite-difference grid. If the value of $\xi$ corresponding to the option price we want lies off the finite-difference grid, we set $f_{1}(\xi, \tau)=0$. We then convert back to financial variables.

The Matlab codes for the finite-difference methods, checks and tables are available at http://www.mth.kcl.ac.uk/ shaww/web_page/papers/asians/ 